\title{
Existence and Numerical Simulation of Solutions for Fractional Equations Involving Two Fractional Orders with Nonlocal Boundary Conditions
}

\author{
Jing Zhao, Peifen Lu, and Yiliang Liu \\ Guangxi Key Laboratory of Hybrid Computation and IC Design Analysis and College of Sciences, Guangxi University for Nationalities, \\ Nanning, Guangxi 530006, China
}

Correspondence should be addressed to Yiliang Liu; yiliangliu100@126.com

Received 26 March 2013; Accepted 6 June 2013

Academic Editor: Naseer Shahzad

Copyright (c) 2013 Jing Zhao et al. This is an open access article distributed under the Creative Commons Attribution License, which permits unrestricted use, distribution, and reproduction in any medium, provided the original work is properly cited.

We study a boundary value problem for fractional equations involving two fractional orders. By means of a fixed point theorem, we establish sufficient conditions for the existence and uniqueness of solutions for the fractional equations. In addition, we describe the dynamic behaviors of the fractional Langevin equation by using the $G_{2}$ algorithm.

\section{Introduction}

Fractional differential equations arise in many engineering and scientific disciplines as the mathematical modeling of systems and processes in various fields, such as physics, mechanics, chemical technology, population dynamics, biotechnology, and economics (see, e.g., [1-7]). As one of the important topics in the research on differential equations, the boundary value problem has attained a great deal of attention from many researchers (see [8-18]) and the references therein. As pointed out in [19], the nonlocal boundary condition can be more useful than the standard condition to describe some physical phenomena. There are several noteworthy papers (see [20-22]) dealing with nonlocal boundary value problems of fractional differential equations.

In [19], Benchohra et al. investigated the existence and uniqueness of the solutions for the differential equations with nonlocal conditions:

$$
\begin{array}{cl}
{ }^{c} D^{\alpha} u(t)+f(t, u(t))=0, & 1<\alpha \leq 2,0<t<T, \\
u(0)=g(u), & u(T)=u_{T},
\end{array}
$$

where ${ }^{c} D^{\alpha}$ denotes Caputo's fractional derivative of order $\alpha$ with the lower limit zero.
In [22], Zhong and Lin studied the existence and uniqueness of solutions in the nonlocal and multiple-point boundary value problem for fractional differential equation:

$$
\begin{aligned}
& { }^{c} D^{q} u(t)+f(t, u(t))=0, \quad 0<t<1,1<q \leq 2, \\
& u(0)=u_{0}+g(u), \quad u^{\prime}(1)=u_{1}+\sum_{i=1}^{m-2} b_{i} u^{\prime}\left(\xi_{i}\right),
\end{aligned}
$$

where ${ }^{c} D^{q}$ denotes Caputo's fractional derivative of order $q$ with the lower limit zero.

In this paper we will study the fractional Langevin equation where the fractional derivative is in Caputo sense. In 1908 the French physicist Langevin introduced the concept of the equation of motion with a random variable, which reads as

$$
m \frac{d^{2} x(t)}{d t^{2}}=-\gamma \frac{d x(t)}{d t}+F(t)+\xi(t)
$$

where $m$ is the mass of the particle, $\gamma$ is the coefficient of viscosity, $F(x)$ is the external force, and $\xi(t)$ is the random force. The Langevin equation is always regarded as the first stochastic differential equation.

Langevin equation has been widely used to describe the evolution of physical phenomena in fluctuating environm- 
ents [23-25]. For instance, Brownian motion is well described by the Langevin equation when the random fluctuation force is assumed to be white noise. In case the random fluctuation force is not white noise, the motion of the particle is described by the generalized Langevin equation [26]. For systems in complex media, ordinary Langevin equation does not provide the correct description of the dynamics. Various generalizations of Langevin equations have been proposed to describe dynamical processes in a fractal medium. One such generalization is the generalized Langevin equation [27-32] which incorporates the fractal and memory properties with a dissipative memory kernel into the Langevin equation.

Fractional order models are more accurate than integerorder models as fractional order models allow more degrees of freedom. The presence of memory term in such models not only takes into account the history of the process involved but also carries its impact to present and future development of the process. Fractional differential equations are also regarded as an alternative model to nonlinear differential equations [33]. In consequence, the subject of fractional differential equations is gaining much importance and attention. For some recent work on fractional differential equations, see $[1,34-46]$.

In [47], Ahmad et al. studied nonlinear Langevin equation involving two fractional orders in different intervals:

$$
\begin{gathered}
{ }^{c} D^{\beta}\left({ }^{c} D^{\alpha}+\lambda\right) x(t)=f\left(t, x(t), x^{\prime}(t)\right), \\
0<t<1, \quad 1<\alpha \leq 2, \quad 0<\beta \leq 1, \\
x(0)=0, \quad x(\eta)=0, \quad x(1)=0, \quad 0<\eta<1,
\end{gathered}
$$

where ${ }^{c} D^{\alpha}$ and ${ }^{c} D^{\beta}$ denote Caputo's fractional derivative of order $\alpha$ and $\beta$ with the lower limit zero.

In [48], A. Chen and Y. Chen studied existence of solutions to nonlinear Langevin equation involving two fractional orders with boundary value conditions:

$$
\begin{array}{r}
{ }^{c} D^{\beta}\left({ }^{c} D^{\alpha}+\lambda\right) u(t)=f\left(t, u(t), u^{\prime}(t)\right), \\
0<t<T, \quad 0<\alpha \leq 1, \quad 1<\beta \leq 2, \\
u(0)=-u(T), \quad u^{\prime}(0)=u^{\prime}(T)=0,
\end{array}
$$

where ${ }^{c} D^{\alpha}$ and ${ }^{c} D^{\beta}$ denote Caputo's fractional derivative of order $\alpha$ and $\beta$ with the lower limit zero.

The fractional calculus has been studied for more than three hundred years. In recent few decades, the fractional calculus has been widely used in many fields such as chaotic dynamics, viscoelasticity, acoustics, and physical chemistry. In [49], Guo studied the numerical solution of fractional partial differential equations. In [50], Guo studied the numerical simulation of the fractional Langevin equation.

As far as we know, there are no papers discussing the existence and numerical simulation of solutions for fractional equations involving two fractional orders with nonlocal boundary conditions.

Motivated by the works mentioned above, in this paper, we establish the existence and uniqueness of solutions by the fixed point theorem and use $G_{2}$ algorithm to describe the dynamic behaviors for the following problem:

$$
\begin{gathered}
{ }^{c} D^{\beta}\left({ }^{c} D^{\alpha}+\lambda\right) u(t)=f\left(t, u(t), u^{\prime}(t)\right), \\
0<t<1, \quad 1<\alpha \leq 2, \quad 0<\beta \leq 1, \\
\alpha_{1} u(0)+\beta_{1} u(1)=g_{1}(u), \\
\alpha_{2} u^{\prime}(0)+\beta_{2} u^{\prime}(1)=g_{2}(u), \\
u(0)=\eta u^{\prime}(0), \quad \eta \neq 0,
\end{gathered}
$$

where ${ }^{c} D^{\alpha}$ and ${ }^{c} D^{\beta}$ denote Caputo's fractional derivative of order $\alpha$ and $\beta$ with the lower limit zero, $f:[0,1] \times R^{2} \rightarrow R$ is a given continuous function and $\lambda$ is a real number, and $g_{1}, g_{2}: C([0,1], R) \rightarrow R$ are two continuous functions, $\alpha \beta_{2}\left[\left(\alpha_{1}+\beta_{1}\right) \eta+\beta_{1}\right] \neq \beta_{1}\left(\alpha_{2}+\beta_{2}\right)$. Evidently, problem (6) not only includes boundary value problems mentioned above but also extends them to a much wider case.

The organization of this paper is as follows. In Section 2, we will give some lemmas which are essential to prove our main results. In Section 3, main results are given. In Section 4, we will give the numerical simulation for the fractional Langevin equation.

\section{Preliminaries}

In this section, we introduce notations, definitions, and preliminary facts. Throughout this paper, set $\mathscr{C}=C([0,1], R)$ denotes the Banach space of all continuous functions from $[0,1] \rightarrow R$ with the norm $\|x\|_{\mathscr{C}}=\sup _{t \in[0,1]}|x(t)|$. We also introduce the Banach space $u \in C^{1}([0,1], R)$ endowed with the norm defined by $\|u\|_{C^{1}}=\max \left\{\sup _{t \in[0,1]}|u(t)|\right.$, $\left.\sup _{t \in[0,1]}\left|u^{\prime}(t)\right|\right\}$.

For the convenience of the readers, let us recall the following useful definitions and fundamental facts of fractional calculus theory.

Definition 1 (see $[1,6]$ ). The Riemann-Liouville derivative of order $\gamma$ with the lower limit zero for a function $f:[0, \infty) \rightarrow$ $R$ can be written as

$$
\begin{array}{r}
{ }^{L} D^{\gamma} f(t)=\frac{1}{\Gamma(n-\gamma)} \frac{d^{n}}{d t^{n}} \int_{0}^{t} \frac{f(s)}{(t-s)^{\gamma+1-n}} d s, \\
t>0, \quad n-1<\gamma<n .
\end{array}
$$

Definition 2 (see $[1,6]$ ). The fractional (arbitrary) order integral of the function $f:[0, \infty) \rightarrow R$ of order $p>0$ is defined by

$$
I^{p} f(x)=\frac{1}{\Gamma(p)} \int_{0}^{x}(x-s)^{p-1} f(s) d s .
$$

Definition 3 (see [1]). Let $\alpha \geq 0, n=[\alpha]+1$. If $f \in A C^{n}[a, b]$, the Caputo fractional derivative of order $\alpha$ of $f$ is defined by

$$
\begin{array}{r}
{ }^{c} D^{\alpha} f(t)=\frac{1}{\Gamma(n-\alpha)} \int_{a}^{t} \frac{f^{(n)}(s)}{(t-s)^{\alpha+1-n}} d s, \\
t>0, \quad n-1<\alpha<n .
\end{array}
$$


Definition 4 (see [6]). Let $p \in(n-1, n], n \in N$ and the the Grünwald-Letnikov fractional derivative of order $p$ of $f$ defined by

$$
D^{p} f(t)=\lim _{\substack{h \rightarrow 0 \\
n h=t-a}} \frac{1}{h^{p}} \sum_{r=0}^{n}(-1)^{r}\left(\begin{array}{l}
p \\
r
\end{array}\right) f(t-r h),
$$

where $\left(\begin{array}{c}p \\ r\end{array}\right)=p(p-1)(p-2) \cdots(p-r+1) / r$ !.

Lemma 5 (see [1]). Let $p \in(m-1, m], m \in N$ and the Caputo derivative of order $p$ for a function $f:[0, \infty) \rightarrow R$. If for $t \in[0,1], f \in A C^{m}[0,1]$ or $f \in C^{m}[0,1]$,

$$
I^{p c} D^{p} f(t)=f(t)-\sum_{k=0}^{m-1} \frac{t^{k}}{k !} f^{(k)}(0) .
$$

We also easily prove the following lemmas.

Lemma 6. Let $\sigma \in L^{q}([0,1], R), q>1 /(\alpha+\beta) .{ }^{c} D^{\alpha} u \in$ $C^{1}([0,1], R), u \in C^{2}([0,1], R)$ satisfying the following differential equation:

$$
\begin{gathered}
{ }^{c} D^{\beta}\left({ }^{c} D^{\alpha}+\lambda\right) u(t)=\sigma(t), \\
0<t<1, \quad 1<\alpha \leq 2, \quad 0<\beta \leq 1, \\
\alpha_{1} u(0)+\beta_{1} u(1)=g_{1}(u), \\
\alpha_{2} u^{\prime}(0)+\beta_{2} u^{\prime}(1)=g_{2}(u), \\
u(0)=\eta u^{\prime}(0), \quad \eta \neq 0,
\end{gathered}
$$

is a solution of the following integral equation:

$u(t)$

$$
\begin{aligned}
& =\frac{1}{\Gamma(\alpha)} \int_{0}^{t}(t-\tau)^{\alpha-1} \\
& \quad \times\left[\frac{1}{\Gamma(\beta)} \int_{0}^{\tau}(\tau-s)^{\beta-1} \sigma(s) d s-\lambda u(\tau)\right] d \tau \\
& +\frac{\alpha \beta_{2}(\eta+t)-t^{\alpha}\left(\alpha_{2}+\beta_{2}\right)}{\alpha \beta_{2}\left[\left(\alpha_{1}+\beta_{1}\right) \eta+\beta_{1}\right]-\beta_{1}\left(\alpha_{2}+\beta_{2}\right)} g_{1}(u) \\
& -\frac{\beta_{1}(\eta+t)-t^{\alpha}\left[\left(\alpha_{1}+\beta_{1}\right) \eta+\beta_{1}\right]}{\alpha \beta_{2}\left[\left(\alpha_{1}+\beta_{1}\right) \eta+\beta_{1}\right]-\beta_{1}\left(\alpha_{2}+\beta_{2}\right)} g_{2}(u) \\
& +\frac{\beta_{1} t^{\alpha}\left(\alpha_{2}+\beta_{2}\right)-\alpha \beta_{1} \beta_{2}(\eta+t)}{\Gamma(\alpha)\left[\alpha \beta_{2}\left(\left(\alpha_{1}+\beta_{1}\right) \eta+\beta_{1}\right)-\beta_{1}\left(\alpha_{2}+\beta_{2}\right)\right]} \\
& \quad \times \int_{0}^{1}(1-\tau)^{\alpha-1} \\
& \quad \times\left[\frac{1}{\Gamma(\beta)} \int_{0}^{\tau}(\tau-s)^{\beta-1} \sigma(s) d s-\lambda u(\tau)\right] d \tau
\end{aligned}
$$

$$
\begin{aligned}
& +\frac{\beta_{2}\left[\beta_{1}(\eta+t)-t^{\alpha}\left(\left(\alpha_{1}+\beta_{1}\right) \eta+\beta_{1}\right)\right]}{\Gamma(\alpha-1)\left[\alpha \beta_{2}\left(\left(\alpha_{1}+\beta_{1}\right) \eta+\beta_{1}\right)-\beta_{1}\left(\alpha_{2}+\beta_{2}\right)\right]} \\
& \times \int_{0}^{1}(1-\tau)^{\alpha-2} \\
& \quad \times\left[\frac{1}{\Gamma(\beta)} \int_{0}^{\tau}(\tau-s)^{\beta-1} \sigma(s) d s-\lambda u(\tau)\right] d \tau .
\end{aligned}
$$

Proof. According to Lemma 5 and applying the operator $I^{\beta}$ to both sides of (12), for some constants $c_{0}, c_{1}$, and $c_{2}$, we get

$$
I^{\beta c} D^{\beta}\left({ }^{c} D^{\alpha}+\lambda\right) u(t)=I^{\beta} \sigma(t) ;
$$

then the above equation can be written as

$$
\left({ }^{c} D^{\alpha}+\lambda\right) u(t)=I^{\beta} \sigma(t)+c_{0},
$$

and applying the operator $I^{\alpha}$ to both sides of the above equation, we obtain

$$
I^{\alpha} D^{\alpha} u(t)=I^{\alpha}\left[I^{\beta} \sigma(t)-\lambda u(t)\right]+I^{\alpha} c_{0}
$$

then the above equation can be written as

$$
u(t)=I^{\alpha}\left[I^{\beta} \sigma(t)-\lambda u(t)\right]+I^{\alpha} c_{0}+c_{1}+c_{2} t
$$

that can be written as (13). The proof is completed.

Definition 7. The function $u \in C^{1}([0,1], R)$ satisfying (13) is a generalized solution of the nonlocal boundary value problem (6).

Lemma 8 (Krasnoselskii). Let $\mathfrak{B}$ be a closed convex and nonempty subset of $X$. Suppose that $\mathscr{L}$ and $\mathcal{N}$ are general nonlinear operators which map $\mathfrak{B}$ into $X$ such that

(1) $\mathscr{L} x+\mathscr{N} y \in \mathfrak{B}$ whenever $x, y \in \mathfrak{B}$;

(2) $\mathscr{L}$ is a contraction mapping;

(3) $\mathcal{N}$ is compact and continuous.

Then there exists $z \in \mathfrak{B}$ such that $z=\mathscr{L} z+\mathscr{N} z$.

\section{Main Results}

In order to apply Lemma 8 to prove our main results, we first give $F, S, T$ as follows. Let $\bar{\Omega}_{r}=\left\{u \in C^{1}([0,1], R):\|u\|_{C^{1}} \leq\right.$ $r\}, r>0$. 
Define an operator $F: C^{1} \rightarrow C^{1}$ by

$$
(F u)(t)=(S u)(t)+(T u)(t),
$$

$(\mathrm{Su})(t)$

$$
\begin{aligned}
=\frac{1}{\Gamma(\alpha)} \int_{0}^{t}(t-\tau)^{\alpha-1} & \\
\times & {\left[\frac{1}{\Gamma(\beta)} \int_{0}^{\tau}(\tau-s)^{\beta-1} f\left(s, u(s), u^{\prime}(s)\right) d s\right.} \\
& \quad-\lambda u(\tau)] d \tau
\end{aligned}
$$

$(\mathrm{Tu})(t)$

$$
\begin{aligned}
& =\frac{\alpha \beta_{2}(\eta+t)-t^{\alpha}\left(\alpha_{2}+\beta_{2}\right)}{\alpha \beta_{2}\left[\left(\alpha_{1}+\beta_{1}\right) \eta+\beta_{1}\right]-\beta_{1}\left(\alpha_{2}+\beta_{2}\right)} g_{1}(u) \\
& -\frac{\beta_{1}(\eta+t)-t^{\alpha}\left[\left(\alpha_{1}+\beta_{1}\right) \eta+\beta_{1}\right]}{\alpha \beta_{2}\left[\left(\alpha_{1}+\beta_{1}\right) \eta+\beta_{1}\right]-\beta_{1}\left(\alpha_{2}+\beta_{2}\right)} g_{2}(u) \\
& +\frac{\beta_{1} t^{\alpha}\left(\alpha_{2}+\beta_{2}\right)-\alpha \beta_{1} \beta_{2}(\eta+t)}{\Gamma(\alpha)\left[\alpha \beta_{2}\left(\left(\alpha_{1}+\beta_{1}\right) \eta+\beta_{1}\right)-\beta_{1}\left(\alpha_{2}+\beta_{2}\right)\right]} \\
& \times \int_{0}^{1}(1-\tau)^{\alpha-1} \\
& \times\left[\frac{1}{\Gamma(\beta)} \int_{0}^{\tau}(\tau-s)^{\beta-1} f\left(s, u(s), u^{\prime}(s)\right) d s\right. \\
& \quad-\lambda u(\tau)] d \tau \\
& +\frac{\beta_{2}\left[\beta_{1}(\eta+t)-t^{\alpha}\left(\left(\alpha_{1}+\beta_{1}\right) \eta+\beta_{1}\right)\right]}{\Gamma(\alpha-1)\left[\alpha \beta_{2}\left(\left(\alpha_{1}+\beta_{1}\right) \eta+\beta_{1}\right)-\beta_{1}\left(\alpha_{2}+\beta_{2}\right)\right]} \\
& \times \int_{0}^{1}(1-\tau)^{\alpha-2} \\
& \times\left[\frac{1}{\Gamma(\beta)} \int_{0}^{\tau}(\tau-s)^{\beta-1} f\left(s, u(s), u^{\prime}(s)\right) d s\right. \\
& \quad-\lambda u(\tau)] d \tau .
\end{aligned}
$$

Lemma 9. The function $u \in C^{1}([0,1], R)$ is a generalized solution of the nonlocal boundary value problem (6) if $F u(t)=$ $u(t)$, for all $t \in[0,1]$.

Proof. Firstly, we show that $u \in C^{1}$.

Assuming $u \in C^{1}$ is a generalized solution of the problem (6), there exist three constants $c_{0}, c_{1}$, and $c_{2}$. Equation (13) can be written as

$$
\begin{aligned}
u(t)= & I^{\alpha+\beta} f\left(t, u(t), u^{\prime}(t)\right)-\lambda I^{\alpha} u(t) \\
& +\frac{c_{0} t^{\alpha}}{\Gamma(1+\alpha)}+c_{1}+c_{2} t,
\end{aligned}
$$

and differentiating both sides of the above equation, we get

$$
\begin{aligned}
u^{\prime}(t)= & I^{\alpha+\beta-1} f\left(t, u(t), u^{\prime}(t)\right)-\lambda I^{\alpha-1} u(t) \\
& +\frac{c_{0} t^{\alpha-1}}{\Gamma(\alpha)}+c_{2} .
\end{aligned}
$$

It is clear that every term of the above equation belongs to $C$; then $u \in C^{1}$.

Secondly, we show that $u$ is the generalized solution of the problem (6).

Let $u$ be a generalized solution of the problem (6) and

$$
\begin{aligned}
u(t)= & I^{\alpha+\beta} f\left(t, u(t), u^{\prime}(t)\right)-\lambda I^{\alpha} u(t)+\frac{c_{0} t^{\alpha}}{\Gamma(1+\alpha)} \\
& +c_{1}+c_{2} t .
\end{aligned}
$$

Applying the operator ${ }^{c} D^{\alpha}$ to both sides of the above equation, we obtain

$$
\begin{aligned}
{ }^{c} D^{\alpha} u(t)= & { }^{c} D^{\alpha}\left[I^{\alpha+\beta} f\left(t, u(t), u^{\prime}(t)\right)-\lambda I^{\alpha} u(t)\right. \\
& \left.+\frac{c_{0} t^{\alpha}}{\Gamma(1+\alpha)}+c_{1}+c_{2} t\right] \\
= & { }^{c} D^{\alpha} I^{\alpha+\beta} f\left(t, u(t), u^{\prime}(t)\right) \\
& -\lambda^{c} D^{\alpha} I^{\alpha} u(t)+{ }^{c} D^{\alpha} \frac{c_{0} t^{\alpha}}{\Gamma(1+\alpha)} \\
& +{ }^{c} D^{\alpha} c_{1}+{ }^{c} D^{\alpha} c_{2} t \\
= & I^{\beta} f\left(t, u(t), u^{\prime}(t)\right)-\lambda u(t), \\
\left({ }^{c} D^{\alpha}+\lambda\right) u(t)= & I^{\beta} f\left(t, u(t), u^{\prime}(t)\right),
\end{aligned}
$$

and then applying the operator ${ }^{c} D^{\beta}$ to both sides of the above equation, we obtain

$$
\begin{aligned}
{ }^{c} D^{\beta}\left({ }^{c} D^{\alpha}+\lambda\right) u(t) & ={ }^{c} D^{\beta} I^{\beta} f\left(t, u(t), u^{\prime}(t)\right) \\
& =f\left(t, u(t), u^{\prime}(t)\right) .
\end{aligned}
$$

By simple calculations, it is clear that $u$ satisfies conditions (6); then it is a generalized solution for the problem (6). The proof is completed. 
For convenience, let us set

$$
\begin{aligned}
& \Lambda_{1}=\left(\alpha_{1}+\beta_{1}\right) \eta+\beta_{1}, \\
& \Lambda_{2}= \frac{1}{\alpha \beta_{2}\left[\left(\alpha_{1}+\beta_{1}\right) \eta+\beta_{1}\right]-\beta_{1}\left(\alpha_{2}+\beta_{2}\right)}, \\
& \Lambda_{3}=\int_{0}^{1}(1-\tau)^{\alpha-1} \\
& \quad \times\left[\frac{1}{\Gamma(\beta)} \int_{0}^{\tau}(\tau-s)^{\beta-1} f\left(s, u(s), u^{\prime}(s)\right) d s\right. \\
&\quad-\lambda u(\tau)] d \tau,
\end{aligned}
$$$$
\Lambda_{4}=\int_{0}^{1}(1-\tau)^{\alpha-2}
$$$$
\times\left[\frac{1}{\Gamma(\beta)} \int_{0}^{\tau}(\tau-s)^{\beta-1} f\left(s, u(s), u^{\prime}(s)\right) d s\right.
$$$$
-\lambda u(\tau)] d \tau
$$$$
\Lambda_{5}=\int_{0}^{1}(1-\tau)^{\alpha-1}
$$$$
\times\left[\frac{1}{\Gamma(\beta)} \int_{0}^{\tau}(\tau-s)^{\beta-1} f\left(s, v(s), v^{\prime}(s)\right) d s\right.
$$$$
-\lambda v(\tau)] d \tau
$$$$
\Lambda_{6}=\int_{0}^{1}(1-\tau)^{\alpha-2}
$$$$
\times\left[\frac{1}{\Gamma(\beta)} \int_{0}^{\tau}(\tau-s)^{\beta-1} f\left(s, v(s), v^{\prime}(s)\right) d s\right.
$$$$
-\lambda v(\tau)] d \tau
$$

Clearly, for any $t \in[0,1]$,

$$
\begin{aligned}
& (S u)^{\prime}(t) \\
& =\frac{1}{\Gamma(\alpha-1)} \int_{0}^{t}(t-\tau)^{\alpha-2} \\
& \times\left[\frac{1}{\Gamma(\beta)} \int_{0}^{\tau}(\tau-s)^{\beta-1} f\left(s, u(s), u^{\prime}(s)\right) d s\right. \\
& -\lambda u(\tau)] d \tau,
\end{aligned}
$$

$(T u)^{\prime}(t)$

$$
\begin{aligned}
= & \left(\alpha \beta_{2}-\alpha t^{\alpha-1}\left(\alpha_{2}+\beta_{2}\right)\right) \Lambda_{2} g_{1}(u) \\
& +\left(-\beta_{1}+\alpha t^{\alpha-1} \Lambda_{1}\right) \Lambda_{2} g_{2}(u) \\
& +\frac{\left[\alpha \beta_{1} t^{\alpha-1}\left(\alpha_{2}+\beta_{2}\right)-\alpha \beta_{1} \beta_{2}\right] \Lambda_{2} \Lambda_{3}}{\Gamma(\alpha)} \\
& +\frac{\beta_{2}\left(\beta_{1}-\alpha t^{\alpha-1} \Lambda_{1}\right) \Lambda_{2} \Lambda_{4}}{\Gamma(\alpha-1)} .
\end{aligned}
$$

Now, we make the following hypotheses.

(H1) There exist two real-valued functions $g \in L^{1 / r}([0,1]$, $R$ ) for some $r \in(0,1)$, such that

$$
\begin{aligned}
& \left|f\left(t, u, u^{\prime}\right)-f\left(t, v, v^{\prime}\right)\right| \\
& \quad \leq 2 g(t) \max \left\{|u-v|,\left|u^{\prime}-v^{\prime}\right|\right\},
\end{aligned}
$$

for almost all $t \in[0,1], u, v \in R$.

(H2) There exist two positive constants $l_{1}, l_{2}$ such that $l_{1}+$ $l_{2}=L<1$. Moreover, $g_{1}(0)=0, g_{2}(0)=0$ and

$$
\begin{array}{r}
\left|g_{1}(u)-g_{1}(v)\right| \leq l_{1}\|u-v\|_{C^{1}}, \\
\left|g_{2}(u)-g_{2}(v)\right| \leq l_{2}\|u-v\|_{C^{1}}, \\
\forall u, v \in C^{1}([0,1], R) .
\end{array}
$$

Theorem 10. Let $f:[0,1] \times R \times R \rightarrow R$ be a jointly continuous function and the assumptions (H1) and (H2) hold. In addition, assume that

$$
\begin{aligned}
\Lambda \triangleq \max \{ & \Upsilon_{1}+(\eta+1) \\
& \times\left(\alpha \beta_{2} \Lambda_{2} L+\beta_{1} \Lambda_{2} L+\beta_{1} \beta_{2} \Lambda_{2} \Upsilon_{2}\right) \\
& +\beta_{1}\left(\alpha_{2}+\beta_{2}\right) \Lambda_{2} \Upsilon_{1}, \Upsilon_{2} \\
& +\left(\alpha_{2}+\beta_{2}\right) \alpha \beta_{1} \Lambda_{2} \Upsilon_{1}+\alpha \beta_{2} \Lambda_{2} L \\
& \left.+\alpha \Lambda_{1} \Lambda_{2} L+\beta_{1} \beta_{2} \Lambda_{2} \Upsilon_{2}\right\}<1,
\end{aligned}
$$

where $p \in(0,1), 1<\alpha \leq 2,0<\beta \leq 1, \eta \neq 0, g^{*}=$ $\left(\int_{0}^{1}(g(s))^{1 /(1-p)} d s\right)^{1-p}$.

Then the problem (6) has at most one solution.

Proof. The proof will be given in two steps.

Step 1. F is bounded.

Now we show that $F \bar{\Omega}_{r} \subset \bar{\Omega}_{r}$. 
6

Journal of Applied Mathematics

$$
\begin{aligned}
& \left|\Lambda_{3}\right|=\mid \int_{0}^{1}(1-\tau)^{\alpha-1} \\
& \times\left[\frac{1}{\Gamma(\beta)} \int_{0}^{\tau}(\tau-s)^{\beta-1} f\left(s, u(s), u^{\prime}(s)\right) d s\right. \\
& -\lambda u(\tau)] d \tau \\
& \leq \int_{0}^{1}(1-\tau)^{\alpha-1} \\
& \times\left[\frac{1}{\Gamma(\beta)} \int_{0}^{\tau}(\tau-s)^{\beta-1}\right. \\
& \left.\times\left(\int_{0}^{\tau}(g(s))^{1 /(1-p)} d s\right)^{1-p}\right] d \tau \\
& +\frac{M}{\Gamma(\beta)} \int_{0}^{1}(1-\tau)^{\alpha-1} \int_{0}^{\tau}(\tau-s)^{\beta-1} d s d \tau \\
& +|\lambda| r \int_{0}^{1}(1-\tau)^{\alpha-1} d \tau \\
& \leq \frac{2 r g^{*}}{\Gamma(\beta)} \int_{0}^{1}(1-\tau)^{\alpha-1}\left(\frac{p \tau^{(\beta+p-1) / p}}{\beta+p-1}\right)^{p} d \tau \\
& +\frac{M B(\beta+1, \alpha)}{\beta \Gamma(\beta)}+\frac{|\lambda| r}{\alpha} \\
& \leq \frac{2 r g^{*} p^{p} B(\beta+p, \alpha)}{\Gamma(\beta)(\beta+p-1)^{p}}+\frac{M \Gamma(\alpha)}{\Gamma(\alpha+\beta+1)}+\frac{|\lambda| r}{\alpha} \triangleq \Phi_{3} \text {. }
\end{aligned}
$$$$
\times\left(\left|f\left(s, u(s), u^{\prime}(s)\right)-f(s, 0,0)\right|\right.
$$$$
+|f(s, 0,0)|) d s
$$$$
+|\lambda u(\tau)|] d \tau
$$$$
\leq \int_{0}^{1}(1-\tau)^{\alpha-1}
$$$$
\times\left[\frac{1}{\Gamma(\beta)} \int_{0}^{\tau}(\tau-s)^{\beta-1}\right.
$$$$
\times\left(2 g(s) \max \left\{|u(s)|,\left|u^{\prime}(s)\right|\right\}+M\right) d s
$$$$
+|\lambda| r] d \tau
$$$$
\leq \int_{0}^{1}(1-\tau)^{\alpha-1} \frac{1}{\Gamma(\beta)} \int_{0}^{\tau}(\tau-s)^{\beta-1} 2 g(s) r d s d \tau
$$$$
+\int_{0}^{1}(1-\tau)^{\alpha-1} \frac{1}{\Gamma(\beta)} \int_{0}^{\tau}(\tau-s)^{\beta-1} M d s d \tau
$$$$
+\int_{0}^{1}(1-\tau)^{\alpha-1}|\lambda| r d \tau
$$$$
\leq \frac{2 r}{\Gamma(\beta)} \int_{0}^{1}(1-\tau)^{\alpha-1} \int_{0}^{\tau}(\tau-s)^{\beta-1} g(s) d s d \tau
$$$$
+\frac{M}{\Gamma(\beta)} \int_{0}^{1}(1-\tau)^{\alpha-1} \int_{0}^{\tau}(\tau-s)^{\beta-1} d s d \tau
$$$$
+|\lambda| r \int_{0}^{1}(1-\tau)^{\alpha-1} d \tau
$$$$
\leq \frac{2 r}{\Gamma(\beta)} \int_{0}^{1}(1-\tau)^{\alpha-1}
$$$$
\times\left[\left(\int_{0}^{\tau}(\tau-s)^{(\beta-1) / p} d s\right)^{p}\right.
$$

Clearly, we also can get

$$
\begin{aligned}
& \Lambda_{4} \leq \frac{2 r g^{*} p^{p} B(\beta+p, \alpha-1)}{\Gamma(\beta)(\beta+p-1)^{p}}+\frac{M \Gamma(\alpha-1)}{\Gamma(\alpha+\beta)}+\frac{|\lambda| r}{\alpha-1} \triangleq \Phi_{4} \text {, } \\
& |(F u)(t)| \\
& =\mid \frac{1}{\Gamma(\alpha)} \int_{0}^{t}(t-\tau)^{\alpha-1} \\
& \times\left[\frac{1}{\Gamma(\beta)} \int_{0}^{\tau}(\tau-s)^{\beta-1}\right. \\
& \times f\left(s, u(s), u^{\prime}(s)\right) d s \\
& -\lambda u(\tau)] d \tau \\
& +\left(\alpha \beta_{2}(\eta+t)-t^{\alpha}\left(\alpha_{2}+\beta_{2}\right)\right) \Lambda_{2} g_{1}(u) \\
& -\left(\beta_{1}(\eta+t)-t^{\alpha} \Lambda_{1}\right) \Lambda_{2} g_{2}(u) \\
& +\frac{\left(\beta_{1} t^{\alpha}\left(\alpha_{2}+\beta_{2}\right)-\alpha \beta_{1} \beta_{2}(\eta+t)\right) \Lambda_{2} \Lambda_{3}}{\Gamma(\alpha)} \\
& +\frac{\beta_{2}\left[\beta_{1}(\eta+t)-t^{\alpha} \Lambda_{1}\right]}{\Gamma(\alpha-1)} \Lambda_{2} \Lambda_{4} \\
& \leq \frac{1}{\Gamma(\alpha)} \int_{0}^{1}(1-\tau)^{\alpha-1} \\
& \times\left[\frac{1}{\Gamma(\beta)} \int_{0}^{\tau}(\tau-s)^{\beta-1}\right. \\
& \times f\left(s, u(s), u^{\prime}(s)\right) d s \\
& -\lambda u(\tau)] d \tau
\end{aligned}
$$




$$
\begin{aligned}
& +\alpha \beta_{2}(\eta+1) \Lambda_{2} l_{1}\|u\|+\Lambda_{1} \Lambda_{2} l_{2}\|u\| \\
& +\frac{\beta_{1}\left(\alpha_{2}+\beta_{2}\right) \Lambda_{2} \Lambda_{3}}{\Gamma(\alpha)}+\frac{\beta_{2} \beta_{1}(\eta+1)}{\Gamma(\alpha-1)} \Lambda_{2} \Lambda_{4} \\
& \leq \frac{\Lambda_{3}}{\Gamma(\alpha)}+\alpha \beta_{2}(\eta+1) \Lambda_{2} l_{1} r+\Lambda_{1} \Lambda_{2} l_{2} r \\
& +\frac{\beta_{1}\left(\alpha_{2}+\beta_{2}\right) \Lambda_{2} \Lambda_{3}}{\Gamma(\alpha)}+\frac{\beta_{2} \beta_{1}(\eta+1)}{\Gamma(\alpha-1)} \Lambda_{2} \Lambda_{4} \\
& \leq \frac{\Phi_{3}}{\Gamma(\alpha)}+\left(\alpha \beta_{2}(\eta+1)+\Lambda_{1}\right) \Lambda_{2} L r \\
& +\frac{\beta_{1}\left(\alpha_{2}+\beta_{2}\right) \Lambda_{2} \Phi_{3}}{\Gamma(\alpha)}+\frac{\beta_{2} \beta_{1}(\eta+1)}{\Gamma(\alpha-1)} \Lambda_{2} \Phi_{4},
\end{aligned}
$$

$\left|(F u)^{\prime}(t)\right|$

$$
\begin{aligned}
= & \mid \frac{1}{\Gamma(\alpha-1)} \int_{0}^{t}(t-\tau)^{\alpha-2} \\
& \times\left[\frac{1}{\Gamma(\beta)} \int_{0}^{\tau}(\tau-s)^{\beta-1}\right. \\
& \times f\left(s, u(s), u^{\prime}(s)\right) d s \\
& \times\left(\alpha \beta_{2}-\alpha t^{\alpha-1}\left(\alpha_{2}+\beta_{2}\right)\right) \Lambda_{2} g_{1}(u) \\
& +\left(-\beta_{1}+\alpha t^{\alpha-1} \Lambda_{1}\right) \Lambda_{2} g_{2}(u) \\
& +\frac{\left[\alpha \beta_{1} t^{\alpha-1}\left(\alpha_{2}+\beta_{2}\right)-\alpha \beta_{1} \beta_{2}\right] \Lambda_{2} \Lambda_{3}}{\Gamma(\alpha)} \\
& +\frac{\beta_{2}\left[\beta_{1}-\alpha t^{\alpha-1} \Lambda_{1}\right] \Lambda_{2} \Lambda_{4}}{\Gamma(\alpha-1)} \mid d \tau \\
+ & \frac{1}{\Gamma(\alpha-1)} \int_{0}^{1}(1-\tau)^{\alpha-2} \\
+ & \times\left[\frac{\alpha \beta_{1}\left(\alpha_{2}+\beta_{2}\right) \Lambda_{2} \Lambda_{3}\|u\|+\alpha \Lambda_{1} \Lambda_{2} l_{2}\|u\|}{\Gamma(\alpha)} \int_{0}^{\tau}(\tau-s)^{\beta-1}\right. \\
& \times f\left(s, u(s), u_{2}^{\prime}(s)\right) d s \\
\Gamma(\alpha-1) & -\lambda u(\tau)] d \tau
\end{aligned}
$$

$$
\begin{aligned}
\leq & \frac{\Lambda_{4}}{\Gamma(\alpha-1)}+\alpha \beta_{2} \Lambda_{2} l_{1} r+\alpha \Lambda_{1} \Lambda_{2} l_{2} r \\
& +\frac{\alpha \beta_{1}\left(\alpha_{2}+\beta_{2}\right) \Lambda_{2} \Lambda_{3}}{\Gamma(\alpha)}+\frac{\beta_{2} \beta_{1} \Lambda_{2} \Lambda_{4}}{\Gamma(\alpha-1)} \\
\leq & \frac{\Phi_{4}}{\Gamma(\alpha-1)}+\alpha\left(\beta_{2}+\Lambda_{1}\right) \Lambda_{2} L r \\
& +\frac{\alpha \beta_{1}\left(\alpha_{2}+\beta_{2}\right) \Lambda_{2} \Phi_{3}}{\Gamma(\alpha)}+\frac{\beta_{2} \beta_{1} \Lambda_{2} \Phi_{4}}{\Gamma(\alpha-1)} .
\end{aligned}
$$

For convenience, we let

$$
\begin{aligned}
\psi=\max \{ & \frac{\Phi_{3}}{\Gamma(\alpha)}+\left(\alpha \beta_{2}(\eta+1)+\Lambda_{1}\right) \Lambda_{2} L r \\
& +\frac{\beta_{1}\left(\alpha_{2}+\beta_{2}\right) \Lambda_{2} \Phi_{3}}{\Gamma(\alpha)} \\
& +\frac{\beta_{2} \beta_{1}(\eta+1)}{\Gamma(\alpha-1)} \Lambda_{2} \Phi_{4}, \frac{\Phi_{4}}{\Gamma(\alpha-1)} \\
& +\alpha\left(\beta_{2}+\Lambda_{1}\right) \Lambda_{2} L r \\
& \left.+\frac{\alpha \beta_{1}\left(\alpha_{2}+\beta_{2}\right) \Lambda_{2} \Phi_{3}}{\Gamma(\alpha)}+\frac{\beta_{2} \beta_{1} \Lambda_{2} \Phi_{4}}{\Gamma(\alpha-1)}\right\},
\end{aligned}
$$

where we have used the Hölder inequality and the following equalities:

$$
B(\beta+1, \alpha)=\int_{0}^{1}(1-\tau)^{\alpha-1} \tau^{\beta} d \tau=\frac{\Gamma(\beta+1) \Gamma(\alpha)}{\Gamma(\alpha+\beta+1)} .
$$

Therefore, $\|(F u)(t)\| \leq \psi$.

Step 2. F is a contraction operator.

For convenience, we get

$$
\begin{aligned}
& |(S u)(t)-(S v)(t)| \\
& =\mid \frac{1}{\Gamma(\alpha)} \int_{0}^{t}(t-\tau)^{\alpha-1} \\
& \quad \times\left[\frac{1}{\Gamma(\beta)} \int_{0}^{\tau}(\tau-s)^{\beta-1} f\left(s, u(s), u^{\prime}(s)\right) d s\right. \\
& \quad-\lambda u(\tau)] d \tau
\end{aligned}
$$




$$
\begin{aligned}
& -\frac{1}{\Gamma(\alpha)} \int_{0}^{t}(t-\tau)^{\alpha-1} \\
& \times\left[\frac{1}{\Gamma(\beta)} \int_{0}^{\tau}(\tau-s)^{\beta-1} f\left(s, v(s), v^{\prime}(s)\right) d s\right. \\
& -\lambda v(\tau)] d \tau \mid \\
& \leq \frac{1}{\Gamma(\alpha)} \int_{0}^{1}(1-\tau)^{\alpha-1} \\
& \times\left[\frac{1}{\Gamma(\beta)} \int_{0}^{\tau}(\tau-s)^{\beta-1} 2 g(s)\right. \\
& \left.\times \max \left\{|u-v|,\left|u^{\prime}-v^{\prime}\right|\right\} d s\right] d \tau \\
& +\frac{|\lambda|}{\Gamma(\alpha)} \int_{0}^{1}(1-\tau)^{\alpha-1}\|u-v\| d \tau \\
& \leq\|u-v\| \\
& \times\left(\frac{1}{\Gamma(\alpha) \Gamma(\beta)} \int_{0}^{1}(1-\tau)^{\alpha-1} \int_{0}^{\tau}(\tau-s)^{\beta-1} 2 g(s) d s d \tau\right. \\
& \left.+\frac{|\lambda|}{\Gamma(\alpha)} \int_{0}^{1}(1-\tau)^{\alpha-1} d \tau\right) \\
& \leq\|u-v\| \\
& \times\left[\frac{2}{\Gamma(\alpha) \Gamma(\beta)} \int_{0}^{1}(1-\tau)^{\alpha-1}\left(\int_{0}^{\tau}(\tau-s)^{(\beta-1) / p} d s\right)^{p}\right. \\
& \left.\times\left(\int_{0}^{\tau}(g(s))^{1 /(1-p)} d s\right)^{1-p} d \tau+\frac{|\lambda|}{\Gamma(\alpha+1)}\right] \\
& \leq\|u-v\|\left(\frac{2 g^{*} p^{p} \Gamma(\beta+p)}{\Gamma(\beta) \Gamma(\alpha+\beta+p)(\beta+p-1)^{p}}+\frac{|\lambda|}{\Gamma(\alpha+1)}\right) \\
& \triangleq \Upsilon_{1}\|u-v\| .
\end{aligned}
$$

Clearly, we can also get

$$
\begin{aligned}
\left|(S u)^{\prime}(t)-(S v)^{\prime}(t)\right| & \\
\leq\|u-v\|( & \frac{2 g^{*} p^{p} \Gamma(\beta+p)}{\Gamma(\beta) \Gamma(\alpha+\beta+p-1)(\beta+p-1)^{p}} \\
& \left.\quad+\frac{|\lambda|}{\Gamma(\alpha)}\right) \triangleq \Upsilon_{2}\|u-v\| .
\end{aligned}
$$

For $u, v \in C^{1}([0,1], R)$ and for each $t \in[0,1]$, we obtain

$$
\begin{aligned}
& |(F u)(t)-(F v)(t)| \\
& =\mid \operatorname{Su}(t)+\left(\alpha \beta_{2}(\eta+t)-t^{\alpha}\left(\alpha_{2}+\beta_{2}\right)\right) \Lambda_{2} g_{1}(u) \\
& -\left(\beta_{1}(\eta+t)-t^{\alpha} \Lambda_{1}\right) \Lambda_{2} g_{2}(u) \\
& +\frac{\left(\beta_{1} t^{\alpha}\left(\alpha_{2}+\beta_{2}\right)-\alpha \beta_{1} \beta_{2}(\eta+t)\right) \Lambda_{2} \Lambda_{3}}{\Gamma(\alpha)} \\
& +\frac{\beta_{2}\left[\beta_{1}(\eta+t)-t^{\alpha} \Lambda_{1}\right]}{\Gamma(\alpha-1)} \Lambda_{2} \Lambda_{4} \\
& -\left[S v(t)+\left(\alpha \beta_{2}(\eta+t)-t^{\alpha}\left(\alpha_{2}+\beta_{2}\right)\right) \Lambda_{2} g_{1}(v)\right. \\
& -\left(\beta_{1}(\eta+t)-t^{\alpha} \Lambda_{1}\right) \Lambda_{2} g_{2}(v) \\
& +\frac{\left(\beta_{1} t^{\alpha}\left(\alpha_{2}+\beta_{2}\right)-\alpha \beta_{1} \beta_{2}(\eta+t)\right) \Lambda_{2} \Lambda_{3}}{\Gamma(\alpha)} \\
& \left.+\frac{\beta_{2}\left[\beta_{1}(\eta+t)-t^{\alpha} \Lambda_{1}\right]}{\Gamma(\alpha-1)} \Lambda_{2} \Lambda_{4}\right] \\
& \leq|(S u)(t)-(S v)(t)| \\
& +\left|\left(\alpha \beta_{2}(\eta+t)-t^{\alpha}\left(\alpha_{2}+\beta_{2}\right)\right)\right| \Lambda_{2} l_{1}\|u-v\| \\
& +\left|\left(\beta_{1}(\eta+t)-t^{\alpha} \Lambda_{1}\right)\right| \Lambda_{2} l_{2}\|u-v\| \\
& +\mid \frac{\left(\beta_{1} t^{\alpha}\left(\alpha_{2}+\beta_{2}\right)-\alpha \beta_{1} \beta_{2}(\eta+t)\right) \Lambda_{2}\left(\Lambda_{3}-\Lambda_{5}\right)}{\Gamma(\alpha)} \\
& +\frac{\beta_{2}\left[\beta_{1}(\eta+t)-t^{\alpha} \Lambda_{1}\right]}{\Gamma(\alpha-1)} \Lambda_{2}\left(\Lambda_{4}-\Lambda_{6}\right) \\
& \leq|(S u)(t)-(S v)(t)| \\
& +\alpha \beta_{2}(\eta+t) \Lambda_{2} l_{1}\|u-v\| \\
& +\beta_{1}(\eta+t) \Lambda_{2} l_{2}\|u-v\| \\
& +\beta_{1} t^{\alpha}\left(\alpha_{2}+\beta_{2}\right) \Lambda_{2} \Upsilon_{1}\|u-v\| \\
& +\beta_{2} \beta_{1}(\eta+t) \Lambda_{2} \Upsilon_{2}\|u-v\| \\
& \leq\|u-v\|\left(\Upsilon_{1}+(\eta+1)\right. \\
& \times\left(\alpha \beta_{2} \Lambda_{2} L+\beta_{1} \Lambda_{2} L+\beta_{1} \beta_{2} \Lambda_{2} \Upsilon_{2}\right) \\
& \left.+\beta_{1}\left(\alpha_{2}+\beta_{2}\right) \Lambda_{2} \Upsilon_{1}\right) \text {, } \\
& \left|(F u)^{\prime}(t)-(F v)^{\prime}(t)\right| \\
& \leq\left|(S u)^{\prime}(t)-(S u)^{\prime}(t)\right| \\
& +\mid\left(\alpha \beta_{2}-\alpha t^{\alpha-1}\left(\alpha_{2}+\beta_{2}\right)\right) \Lambda_{2}\left(g_{1}(u)-g_{1}(v)\right) \\
& +\left(-\beta_{1}+\alpha t^{\alpha-1} \Lambda_{1}\right) \Lambda_{2}\left(g_{2}(u)-g_{2}(v)\right) \mid
\end{aligned}
$$




$$
\begin{aligned}
& +\mid \frac{\left[\alpha \beta_{1} t^{\alpha-1}\left(\alpha_{2}+\beta_{2}\right)-\alpha \beta_{1} \beta_{2}\right] \Lambda_{2}\left(\Lambda_{3}-\Lambda_{5}\right)}{\Gamma(\alpha)} \\
& +\frac{\beta_{2}\left[\beta_{1}-\alpha t^{\alpha-1} \Lambda_{1}\right] \Lambda_{2}\left(\Lambda_{4}-\Lambda_{6}\right)}{\Gamma(\alpha-1)} \mid \\
& \leq \Upsilon_{2}\|u-v\|+\alpha \beta_{2} \Lambda_{2} l_{1}\|u-v\| \\
& +\alpha \Lambda_{1} \Lambda_{2} l_{2}\|u-v\| \\
& +\alpha \beta_{1} t^{\alpha-1}\left(\alpha_{2}+\beta_{2}\right) \Lambda_{2} \Upsilon_{1}\|u-v\| \\
& +\beta_{2} \beta_{1} \Lambda_{2} \Upsilon_{2}\|u-v\| \\
& \leq\|u-v\|\left(\Upsilon_{2}+\left(\alpha_{2}+\beta_{2}\right) \alpha \beta_{1} \Lambda_{2} \Upsilon_{1}\right. \\
& \left.\quad+\alpha \beta_{2} \Lambda_{2} L+\alpha \Lambda_{1} \Lambda_{2} L+\beta_{1} \beta_{2} \Lambda_{2} \Upsilon_{2}\right) .
\end{aligned}
$$

Since $\Lambda<1$, we have $\|F(u)-F(v)\| \leq \Lambda\|u-v\|$; that is, $F$ is a nonlinear contraction. Hence, by using Lemma 8 , the conclusion of the theorem holds by Banach fixed point theorem.

The proof is completed.

Theorem 11. Let $f:[0,1] \times R \times R \rightarrow R$ be a jointly continuous function and the assumptions (H1) and (H2) hold. In addition,

(H3) assume that there exist a constant $l \in(0,1)$ and a realvalued function $m \in L^{1 / l}\left([0,1], R^{+}\right)$such that

$$
\begin{array}{r}
\left|f\left(t, u, u^{\prime}\right)\right| \leq m(t), \quad \forall\left(t, u, u^{\prime}\right) \in[0,1] \times R \times R, \\
\text { with } \sup _{t \in[0,1]}|m(t)|=\|m\| .
\end{array}
$$

Then the problem (6) has at least one solution on $[0,1]$ if

$$
\begin{aligned}
\xi \triangleq \max & \left\{(\eta+1)\left(\alpha \beta_{2} \Lambda_{2} L+\beta_{1} \Lambda_{2} L+\beta_{1} \beta_{2} \Lambda_{2} \Upsilon_{2}\right)\right. \\
& +\beta_{1}\left(\alpha_{2}+\beta_{2}\right) \Lambda_{2} \Upsilon_{1}, \\
& \left(\alpha_{2}+\beta_{2}\right) \alpha \beta_{1} \Lambda_{2} \Upsilon_{1}+\alpha \beta_{2} \Lambda_{2} L \\
& \left.+\alpha \Lambda_{1} \Lambda_{2} L+\beta_{1} \beta_{2} \Lambda_{2} \Upsilon_{2}\right\}<1 .
\end{aligned}
$$

Proof. Step 1. There exists a positive constant $r>0$ such that $\mathrm{Su}+\mathrm{Tu} \in \bar{\Omega}_{r}$.

For $u \in \bar{\Omega}_{r}$, by the same arguments of the first step of the proof in Theorem 10, we have $\|S u+T u\| \leq \psi$. In virtue of the definition of $\psi$ and a simple calculation, we obtain

$$
\psi \leq M+\xi r,
$$

where $M$ is a constant. By the assumptions, $\xi<1$. Therefore, there exists a positive constant $r$ large enough such that

$$
\psi \leq M+\xi r<r .
$$

Hence, there exists a positive constant $r$ such that $S u+T u \in$ $\bar{\Omega}_{r}$.

Step 2. $T$ is a contraction operator.
For $u, v \in C^{1}([0,1], R)$ and for each $t \in[0,1]$, we obtain

$$
\begin{aligned}
& |(T u)(t)-(T v)(t)| \\
& \leq\|u-v\| \\
& \quad\left((\eta+1)\left(\alpha \beta_{2} \Lambda_{2} L+\beta_{1} \Lambda_{2} L+\beta_{1} \beta_{2} \Lambda_{2} \Upsilon_{2}\right)\right. \\
& \left.\quad+\beta_{1}\left(\alpha_{2}+\beta_{2}\right) \Lambda_{2} \Upsilon_{1}\right) \\
& \left|(T u)^{\prime}(t)-(T v)^{\prime}(t)\right| \\
& \leq\|u-v\| \\
& \left(\left(\alpha_{2}+\beta_{2}\right) \alpha \beta_{1} \Lambda_{2} \Upsilon_{1}\right. \\
& \left.\quad+\alpha \beta_{2} \Lambda_{2} L+\alpha \Lambda_{1} \Lambda_{2} L+\beta_{1} \beta_{2} \Lambda_{2} \Upsilon_{2}\right) .
\end{aligned}
$$

Since $\xi<1$, we have $\|T(u)-T(v)\| \leq \xi\|u-v\|$; that is, $T$ is a nonlinear contraction.

Step 3. S is continuous and compact.

Firstly, we show that the operator $S$ is continuous. For $\left\{u_{n}\right\} \subset \bar{\Omega}_{r}, u_{0} \in \bar{\Omega}_{r}$ such that $u_{n} \rightarrow u_{0}$ in $\bar{\Omega}_{r}$; then

$$
\begin{aligned}
& \left|S u_{n}(t)-S u_{0}(t)\right| \\
& =\mid \frac{1}{\Gamma(\alpha)} \int_{0}^{t}(t-\tau)^{\alpha-1} \\
& \times\left[\frac{1}{\Gamma(\beta)} \int_{0}^{\tau}(\tau-s)^{\beta-1} f\left(s, u_{n}(s), u_{n}^{\prime}(s)\right) d s\right. \\
& \left.-\lambda u_{n}(\tau)\right] d \tau \\
& -\frac{1}{\Gamma(\alpha)} \int_{0}^{t}(t-\tau)^{\alpha-1} \\
& \times\left[\frac{1}{\Gamma(\beta)} \int_{0}^{\tau}(\tau-s)^{\beta-1}\right. \\
& \times f\left(s, u_{0}(s), u_{0}^{\prime}(s)\right) d s \\
& \left.-\lambda u_{0}(\tau)\right] d \tau \\
& \leq \mid \frac{1}{\Gamma(\alpha)} \int_{0}^{t}(t-\tau)^{\alpha-1} \\
& \times \frac{1}{\Gamma(\beta)} \int_{0}^{\tau}(\tau-s)^{\beta-1} \\
& \times\left[f\left(s, u_{n}(s), u_{n}^{\prime}(s)\right)\right. \\
& \left.-f\left(s, u_{0}(s), u_{0}^{\prime}(s)\right)\right] d s d \tau \\
& +\frac{\lambda}{\Gamma(\alpha)} \int_{0}^{t}(t-\tau)^{\alpha-1}\left(u_{n}(\tau)-u_{0}(\tau)\right) d \tau \mid \\
& \leq \frac{1}{\Gamma(\alpha)} \int_{0}^{t}(t-\tau)^{\alpha-1} \\
& \times \frac{1}{\Gamma(\beta)} \int_{0}^{\tau}(\tau-s)^{\beta-1}
\end{aligned}
$$


10

Journal of Applied Mathematics

$$
\begin{gathered}
\times 2 g(s) \max \left\{\left|u_{n}(s)-u_{0}(s)\right|\right. \\
\left.+\frac{|\lambda|}{\Gamma(\alpha)} \int_{0}^{t}(t-\tau)^{\alpha-1}\left|u_{n}^{\prime}(s)-u_{0}^{\prime}(s)\right|\right\} d s d \tau \\
\leq \frac{1}{\Gamma(\alpha)} \int_{0}^{t}(t-\tau)^{\alpha-1} \\
\quad \times \frac{1}{\Gamma(\beta)} \int_{0}^{\tau}(\tau-s)^{\beta-1} 2 g(s)\left\|u_{n}-u_{0}\right\| d s d \tau \\
+\frac{|\lambda|}{\Gamma(\alpha)} \int_{0}^{t}(t-\tau)^{\alpha-1}\left\|u_{n}-u_{0}\right\| d \tau \\
\leq\left\|u_{n}-u_{0}\right\|\left(\frac{2}{\Gamma(\alpha)} \int_{0}^{1}(1-\tau)^{\alpha-1} \frac{1}{\Gamma(\beta)}\right. \\
\leq \Upsilon_{1}\left\|u_{n}-u_{0}\right\| \cdot\left(\tau \int_{0}^{\tau}(\tau-s)^{\beta-1} g(s) d s d \tau\right. \\
\left.+\frac{|\lambda|}{\Gamma(\alpha+1)}\right)
\end{gathered}
$$

Similarly, we get

$$
\left|S u_{n}^{\prime}(t)-S u_{0}^{\prime}(t)\right| \leq \Upsilon_{2}\left\|u_{n}-u_{0}\right\|
$$

we get sequences $u_{n}(t)$ and $u_{0}(t)$, which converge on $[0,1]$ with $\lim _{n \rightarrow \infty} u_{n}(t)=u_{0}(t)$ and $\lim _{n \rightarrow \infty} u_{n}^{\prime}(t)=u_{0}^{\prime}(t)$.

Since

$$
\begin{aligned}
& \left\|S u_{n}-S u_{0}\right\| \\
& =\max \left\{\sup _{t \in[0,1]}\left|S u_{n}(t)-S u_{0}(t)\right|,\right. \\
& \left.\sup _{t \in[0,1]}\left|\left(S u_{n}\right)^{\prime}(t)-\left(S u_{0}\right)^{\prime}(t)\right|\right\} .
\end{aligned}
$$

Combining (41) and (42), we can get $\left\|S u_{n}-S u_{0}\right\| \rightarrow 0$. Thus $S$ is continuous in $C^{1}([0,1], R)$.

Secondly, we show that the operator $S$ is equicontinuous. Let $M_{r}=\max _{s \in[0,1]}\left\{\left|f\left(s, u, u^{\prime}\right)\right|, u \in \bar{\Omega}_{r}\right\}$. For any $u \in \bar{\Omega}_{r}$, for all $s_{1}, s_{2} \in[0,1], 0 \leq s_{1}<s_{2} \leq 1$, we obtain

$$
\begin{aligned}
\mid(S u)\left(s_{2}\right)- & (S u)\left(s_{1}\right) \mid \\
=\mid \frac{1}{\Gamma(\alpha)} \int_{0}^{s_{2}}\left(s_{2}-\tau\right)^{\alpha-1} & \\
\times & {\left[\frac{1}{\Gamma(\beta)} \int_{0}^{\tau}(\tau-s)^{\beta-1} f\left(s, u(s), u^{\prime}(s)\right) d s\right.}
\end{aligned}
$$

$$
\begin{aligned}
& -\lambda u(\tau)] d \tau \\
& -\frac{1}{\Gamma(\alpha)} \int_{0}^{s_{1}}\left(s_{1}-\tau\right)^{\alpha-1} \\
& \times\left[\frac{1}{\Gamma(\beta)} \int_{0}^{\tau}(\tau-s)^{\beta-1} f\left(s, u(s), u^{\prime}(s)\right) d s\right. \\
& -\lambda u(\tau)] d \tau \mid \\
& \leq \mid \frac{1}{\Gamma(\alpha)} \int_{0}^{s_{1}}\left[\left(s_{2}-\tau\right)^{\alpha-1}-\left(s_{1}-\tau\right)^{\alpha-1}\right] \\
& \times\left[\frac{1}{\Gamma(\beta)} \int_{0}^{\tau}(\tau-s)^{\beta-1}\left|f\left(s, u(s), u^{\prime}(s)\right)\right| d s\right. \\
& +|\lambda||u(\tau)|] d \tau \\
& +\frac{1}{\Gamma(\alpha)} \int_{s_{1}}^{s_{2}}\left(s_{1}-\tau\right)^{\alpha-1} \\
& \times\left[\frac{1}{\Gamma(\beta)} \int_{0}^{\tau}(\tau-s)^{\beta-1}\left|f\left(s, u(s), u^{\prime}(s)\right)\right| d s\right. \\
& +|\lambda||u(\tau)|] d \tau \mid \\
& \leq \frac{1}{\Gamma(\alpha)} \int_{0}^{s_{1}}\left[\left(s_{2}-\tau\right)^{\alpha-1}-\left(s_{1}-\tau\right)^{\alpha-1}\right] \\
& \times\left(\frac{1}{\Gamma(\beta)} \int_{0}^{\tau}(\tau-s)^{\beta-1} M_{r} d s\right) d \tau \\
& +\frac{1}{\Gamma(\alpha)} \int_{0}^{s_{1}}\left[\left(s_{2}-\tau\right)^{\alpha-1}-\left(s_{1}-\tau\right)^{\alpha-1}\right]|\lambda| r d \tau \\
& +\frac{1}{\Gamma(\alpha)} \int_{s_{1}}^{s_{2}}\left(s_{1}-\tau\right)^{\alpha-1}\left(\frac{1}{\Gamma(\beta)} \int_{0}^{\tau}(\tau-s)^{\beta-1} M_{r} d s\right) d \tau \\
& +\frac{1}{\Gamma(\alpha)} \int_{s_{1}}^{s_{2}}\left(s_{1}-\tau\right)^{\alpha-1}|\lambda| r d \tau \\
& \leq \frac{1}{\Gamma(\alpha)} \int_{0}^{s_{1}}\left[\left(s_{2}-\tau\right)^{\alpha-1}-\left(s_{1}-\tau\right)^{\alpha-1}\right] \\
& \times\left(\frac{M_{r}}{\Gamma(\beta)} \int_{0}^{\tau}(\tau-s)^{\beta-1} d s\right) d \tau \\
& +\frac{|\lambda| r}{\Gamma(\alpha)} \int_{0}^{s_{1}}\left[\left(s_{2}-\tau\right)^{\alpha-1}-\left(s_{1}-\tau\right)^{\alpha-1}\right] d \tau \\
& +\frac{1}{\Gamma(\alpha)} \int_{s_{1}}^{s_{2}}\left(s_{1}-\tau\right)^{\alpha-1}\left(\frac{M_{r}}{\Gamma(\beta)} \int_{0}^{\tau}(\tau-s)^{\beta-1} d s\right) d \tau \\
& +\frac{|\lambda| r}{\Gamma(\alpha)} \int_{s_{1}}^{s_{2}}\left(s_{1}-\tau\right)^{\alpha-1} d \tau
\end{aligned}
$$




$$
\begin{aligned}
& \leq \frac{M_{r}}{\Gamma(\alpha) \Gamma(\beta+1)} \int_{0}^{s_{1}}\left(s_{2}-s_{1}\right)^{\alpha-1} \tau^{\beta} d \tau \\
& +\frac{|\lambda| r\left(s_{2}^{\alpha}-s_{1}^{\alpha}-\left(s_{2}-s_{1}\right)^{\alpha}\right)}{\Gamma(\alpha+1)} \\
& +\frac{M_{r}}{\Gamma(\alpha) \Gamma(\beta+1)} \int_{s_{1}}^{s_{2}}\left(s_{1}-\tau\right)^{\alpha-1} \tau^{\beta} d \tau \\
& +\frac{|\lambda| r\left(s_{2}-s_{1}\right)^{\alpha}}{\Gamma(\alpha+1)} \\
& \leq \frac{M_{r}\left(s_{2}-s_{1}\right)^{\alpha-1}}{\Gamma(\alpha) \Gamma(\beta+1)} \int_{0}^{1} \tau^{\beta} d \tau \\
& +\frac{|\lambda| r\left(s_{2}^{\alpha}-s_{1}^{\alpha}-\left(s_{2}-s_{1}\right)^{\alpha}+\left(s_{1}-s_{2}\right)^{\alpha}\right)}{\Gamma(\alpha+1)} \\
& +\frac{M_{r}}{\Gamma(\alpha) \Gamma(\beta+1)}\left(\int_{s_{1}}^{s_{2}}\left(s_{1}-\tau\right)^{(\alpha-1) / p} d \tau\right)^{p} \\
& \times\left(\int_{s_{1}}^{s_{2}} \tau^{\beta /(1-p)} d \tau\right)^{1-p} \\
& \leq \frac{M_{r}\left(s_{2}-s_{1}\right)^{\alpha-1}}{\Gamma(\alpha) \Gamma(\beta+2)} \\
& +\left(M_{r}\left[p^{p}\left(s_{1}-s_{2}\right)^{\alpha+p-1}\right]\right. \\
& \left.\times\left[(1-p)^{1-p}\left(s_{2}^{(\beta+1-p) /(1-p)}-s_{1}^{(\beta+1-p) /(1-p)}\right)^{1-p}\right]\right) \\
& \times\left(\Gamma(\alpha) \Gamma(\beta+1)(\alpha+p-1)^{p}(\beta+1-p)^{1-p}\right)^{-1} \\
& +\frac{|\lambda| r\left(s_{2}^{\alpha}-s_{1}^{\alpha}-\left(s_{2}-s_{1}\right)^{\alpha}+\left(s_{1}-s_{2}\right)^{\alpha}\right)}{\Gamma(\alpha+1)} .
\end{aligned}
$$

Clearly, we also easily get

$$
\begin{aligned}
& \left|(S u)^{\prime}\left(s_{2}\right)-(S u)^{\prime}\left(s_{1}\right)\right| \\
& \leq \frac{1}{\Gamma(\alpha-1)} \int_{0}^{s_{1}}\left[\left(s_{2}-\tau\right)^{\alpha-2}-\left(s_{1}-\tau\right)^{\alpha-2}\right] \\
& \quad \times\left(\frac{M_{r} \tau^{\beta}}{\Gamma(\beta+1)}+|\lambda| r\right) d \tau \\
& \quad+\frac{M_{r}\left(s_{2}-s_{1}\right)^{\alpha+\beta-1}}{\Gamma(\alpha+\beta)}+\frac{M_{r}\left(s_{2}-s_{1}\right)^{\alpha-1} s_{1}^{\beta}}{\Gamma(\alpha) \Gamma(\beta+1)} \\
& +\frac{|\lambda| r\left(s_{2}-s_{1}\right)^{\alpha}}{\alpha \Gamma(\alpha-1)} .
\end{aligned}
$$

Obviously the right hand side of the above inequality tends to zero independently of $u \in \bar{\Omega}_{r}$, as $t_{2}-t_{1} \rightarrow 0$; we get that $S$ is relatively compact on $\bar{\Omega}_{r}$. Hence, by the ArzeláAscoli theorem, $S$ is compact on $\bar{\Omega}_{r}$.

Thus, all the assumptions of Lemma 8 are satisfied and the conclusion of Lemma 8 implies that the boundary value problem (6) has at least one solution on $[0,1]$.

The proof is completed.

\section{Algorithm for the Fractional Langevin Equation and Examples}

In this paper, we will give the numerical simulation for the fractional Langevin equation.

The definition of fractional order has many kinds; the different definitions will bring different algorithm forms and will cause different proof of the algorithm stability and different method of accuracy analysis. In the practical application, there are three kinds of fractional derivative definitions, such as Grünwald-Letnikov, Riemann-Liouvlle, and Caputo Fractional derivatives.

Remark 12 (see [51]). For $m-1<\alpha \leq m, m \in N, f(t) \in$ $C^{m}[a, b]$,

$$
{ }^{\mathrm{GL}} D^{\alpha} f(t)={ }^{\mathrm{RL}} D^{\alpha} f(t) .
$$

Remark 13 (see [49]). For $f^{(k)}(a)=0, k=0,1, \ldots, m$,

$$
\begin{aligned}
& { }^{\mathrm{GL}} D^{\alpha} f(t)=\sum_{k=0}^{m-1} \frac{f^{(k)}(a)}{\Gamma(k-a+1)}(t-a)^{k-\alpha} \\
& +{ }_{a}^{c} D^{\alpha} f(t)={ }_{a}^{\mathrm{RL}} D^{\alpha} f(t) .
\end{aligned}
$$

In [52], shifted Grünwald-Letnikov formula is defined by ${ }^{\mathrm{GL}}{ }^{\alpha} f(t)$

$$
=\lim _{h \rightarrow 0} \frac{1}{h^{\alpha}} \sum_{k=0}^{[(t-a) / h+p]} w_{k}^{(\alpha)} f(t-(k-p) h), \quad \alpha>0 .
$$

We get the following approximation:

$$
\begin{aligned}
{ }_{a} D^{\alpha} f(t) & \approx \frac{1}{h^{\alpha}} \sum_{k=0}^{[(t-a) / h+p]} w_{k}^{(\alpha)} f(t-(k-p) h) \\
& :=\left({ }_{a} D^{\alpha} f(t)\right)_{G_{S(p)}} .
\end{aligned}
$$

We put a call shifted Grünwald discrete format, simply “ $G_{S(p)}$ algorithm" for short.

In addition, Oldham and Spanier [53] found the following approximation format in 1974:

$$
\begin{gathered}
{ }_{a} D^{-1} f(t)=\lim _{h \rightarrow 0} h \sum_{j=0}^{[(t-a) / h-1 / 2]} f\left(t-\left(j+\frac{1}{2}\right) h\right), \\
{ }_{a} D^{1} f(t)=\lim _{h \rightarrow 0} h^{-1} \sum_{j=0}^{[(t-a) / h+1 / 2]}(-1)^{j} f\left(t-\left(j-\frac{1}{2}\right) h\right) .
\end{gathered}
$$


The approximation format has the rapid convergence properties. So they put forward an improved GrünwaldLetnikov fractional derivative definition (take $p=\alpha / 2$ to (48)):

$$
\begin{aligned}
{ }_{a} D^{\alpha} f(t)= & \lim _{h \rightarrow 0} \frac{h^{-\alpha}}{\Gamma(-\alpha)} \\
& \times \sum_{j=0}^{[(t-a) / h+\alpha / 2]} \frac{\Gamma(j-\alpha)}{\Gamma(j+1)} f\left(t-\left(j-\frac{1}{2} \alpha\right) h\right) .
\end{aligned}
$$

For $a=0$, the above equation can be written as

$$
\begin{aligned}
{ }_{a} D^{\alpha} f(t)= & \lim _{h \rightarrow 0} \frac{h^{-\alpha}}{\Gamma(-\alpha)} \\
& \times \sum_{j=0}^{[t / h+\alpha / 2]} \frac{\Gamma(j-\alpha)}{\Gamma(j+1)} f\left(t-\left(j-\frac{1}{2} \alpha\right) h\right) .
\end{aligned}
$$

Therefore, they put forward "fractional center difference quotient" approximation format called in general " $G_{2}$ algorithm." mula:

In this paper, we use the three-point interpolation for-

$$
\begin{aligned}
f\left(t-\left(j-\frac{1}{2} \alpha\right) h\right) \approx & \left(\frac{\alpha}{4}+\frac{\alpha^{2}}{8}\right) f(t-(j-1) h) \\
& +\left(1-\frac{\alpha^{2}}{4}\right) f(t-j h) \\
& +\left(\frac{\alpha^{2}}{8}-\frac{\alpha}{4}\right) f(t-(j+1) h) .
\end{aligned}
$$

Then " $G_{2}$ algorithm" can be expressed as:

$$
\begin{aligned}
& \left({ }_{a} D^{\alpha} f\left(t_{n}\right)\right)_{G_{2}} \\
& =h^{-\alpha} \sum_{j=0}^{n-1} w_{j}^{(\alpha)}\left\{f_{n-j}+\frac{1}{4} \alpha\left(f_{n-j+1}-f_{n-j-1}\right)\right. \\
& \left.+\frac{1}{8} \alpha^{2}\left(f_{n-j+1}-2 f_{n-j}+f_{n-j-1}\right)\right\} .
\end{aligned}
$$

Remark 14 (see [49]). $G_{2}$ algorithm is based on GrünwaldLetnikov definition, not only used for numerical calculation of fractional derivative $(\alpha \geq 0)$, but also used for numerical calculation of fractional integral $(\alpha \leq 0)$.

As we all know, the fractional Langevin equation form is

$$
{ }^{c} D^{\beta}\left({ }^{c} D^{\alpha}+\lambda\right) u(t)=f(t)+\xi(t),
$$

where $0 \leq t \leq 1,1<\alpha \leq 2,0<\beta \leq 1, \lambda$ is a constant, $f(t)$ is an external force, and $\xi(t)$ is a random force.

The above equation can be written as

$$
\begin{gathered}
{ }^{c} D^{\beta} u(t)=f(t), \\
{ }^{c} D^{\alpha} v(t)=u(t)-\lambda v(t) .
\end{gathered}
$$

According to $G_{2}$ algorithm, the Caputo fractional derivatives above can be written as

$$
\begin{aligned}
& { }^{c} D^{\beta} u(t) \\
& =\lim _{h \rightarrow 0} \frac{h^{-\beta}}{\Gamma(-\beta)} \sum_{j=0}^{[t / h+\beta / 2]} \frac{\Gamma(j-\beta)}{\Gamma(j+1)} u\left(t-\left(j-\frac{\beta}{2}\right) h\right), \\
& { }^{c} D^{\alpha} u(t) \\
& =\lim _{h \rightarrow 0} \frac{h^{-\alpha}}{\Gamma(-\alpha)} \sum_{j=0}^{[t / h+\alpha / 2]} \frac{\Gamma(j-\alpha)}{\Gamma(j+1)} u\left(t-\left(j-\frac{\alpha}{2}\right) h\right) .
\end{aligned}
$$

The previous equations are approximated by the three-point interpolation formula and can be written as

$$
\begin{aligned}
& { }^{c} D^{\beta} u\left(t_{n}\right) \\
& =h^{-\beta} \sum_{j=0}^{n-1} w_{j}^{(\beta)}\left\{u_{n-j}+\frac{1}{4} \beta\left(u_{n-j+1}-u_{n-j-1}\right)\right. \\
& \left.+\frac{1}{8} \beta^{2}\left(u_{n-j+1}-2 u_{n-j}+u_{n-j-1}\right)\right\}, \\
& { }^{c} D^{\alpha} v\left(t_{n}\right) \quad \\
& =h^{-\alpha} \sum_{j=0}^{n-1} w_{j}^{(\alpha)}\left\{u_{n-j}+\frac{1}{4} \alpha\left(u_{n-j+1}-u_{n-j-1}\right)\right. \\
& \left.+\frac{1}{8} \alpha^{2}\left(u_{n-j+1}-2 u_{n-j}+u_{n-j-1}\right)\right\},
\end{aligned}
$$

where

$$
\begin{gathered}
w_{j}^{(\beta)}=(-1)^{j}\left(\begin{array}{l}
\beta \\
j
\end{array}\right), \quad(j=0,1,2, \ldots), \\
\left(\begin{array}{l}
\beta \\
j
\end{array}\right)=\frac{\beta(\beta-1) \cdots(\beta-j+1)}{j !}, \\
w_{j}^{(\alpha)}=(-1)^{j}\left(\begin{array}{c}
\alpha \\
j
\end{array}\right), \quad(j=0,1,2, \ldots), \\
\left(\begin{array}{c}
\alpha \\
j
\end{array}\right)=\frac{\alpha(\alpha-1) \cdots(\alpha-j+1)}{j !} .
\end{gathered}
$$

With the above algorithm we will give some examples.

Example 1. Consider the following fractional differential equations:

$$
\begin{array}{r}
{ }^{c} D^{0.8}\left({ }^{c} D^{1.5}+0.125\right) u(t)=1.1121 u(t)+t^{2}, \\
0 \leq t \leq 1, \\
u(0)=0, \quad\left({ }^{c} D^{1.5}+0.125\right) u(0)=0 .
\end{array}
$$

Obviously, we get

$$
\begin{gathered}
\left|f\left(t, u(t), u^{\prime}(t)\right)\right| \leq 1.1121\|u\|+1, \\
\left|f\left(t, u(t), u^{\prime}(t)\right)-f\left(t, v(t), v^{\prime}(t)\right)\right| \leq g^{*}\|u-v\| .
\end{gathered}
$$




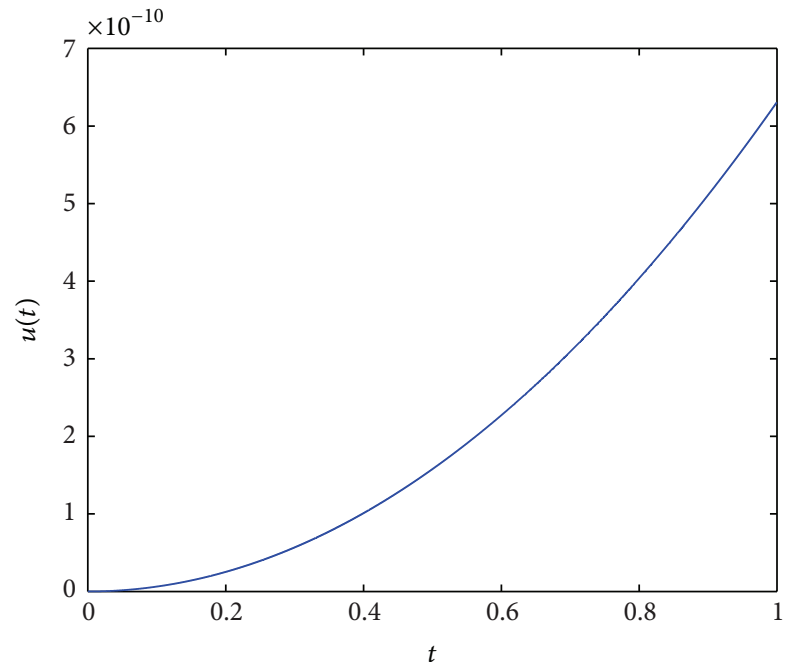

FIGURE 1: The displacement without a Wiener process, the numerical simulation of (60), where $\alpha=1.5, \beta=0.8$.

Letting $p=0.9, g^{*}=0.05, L=l_{1}+l_{2}=0.01, \alpha_{1}=\beta_{1}=\alpha_{2}=$ $\beta_{2}=2, \eta=1.5$, and $g_{1}(u)=g_{2}(u)=0$, we have

$$
\begin{gathered}
\alpha \beta_{2}\left[\left(\alpha_{1}+\beta_{1}\right) \eta+\beta_{1}\right]=24 \neq \beta_{1}\left(\alpha_{2}+\beta_{2}\right)=8 \\
\Lambda \triangleq \max \left\{\Upsilon_{1}+(\eta+1)\right. \\
\quad \times\left(\alpha \beta_{2} L+\beta_{1} \Lambda_{2} L+\beta_{1} \beta_{2} \Lambda_{2} \Upsilon_{2}\right) \\
+\beta_{1}\left(\alpha_{2}+\beta_{2}\right) \Lambda_{2} \Upsilon_{1}, \\
\Upsilon_{2}+\left(\alpha_{2}+\beta_{2}\right)\left(\alpha \Lambda_{2} L+\alpha \beta_{1} \Lambda_{2} \Upsilon_{1}\right) \\
\left.+\alpha \Lambda_{1} \Lambda_{2} L+\beta_{2} \beta_{1} \Lambda_{2} \Upsilon_{2}\right\} \\
=\max \{0.3531,0.3975\}=0.3975<1 .
\end{gathered}
$$

Thus, by Theorem 10, we can get that the problem (60) has at most one solution.

With the above algorithm we get Figures 1 and 2 .

Example 2. Consider the following fractional differential equations:

$$
\begin{aligned}
& { }^{c} D^{0.723}\left({ }^{c} D^{1.625}+0.351\right) u(t) \\
& =1.1121 e^{u(t)}+1.3035 t^{3}, \quad 0 \leq t \leq 1, \\
& u(0)=0, \quad\left({ }^{c} D^{1.625}+0.351\right) u(0)=0 .
\end{aligned}
$$

Obviously, we get

$$
\begin{gathered}
\left|f\left(t, u(t), u^{\prime}(t)\right)\right| \leq\|u\|^{2} \\
\left|f\left(t, u(t), u^{\prime}(t)\right)-f\left(t, v(t), v^{\prime}(t)\right)\right| \leq g^{*}\|u-v\| .
\end{gathered}
$$

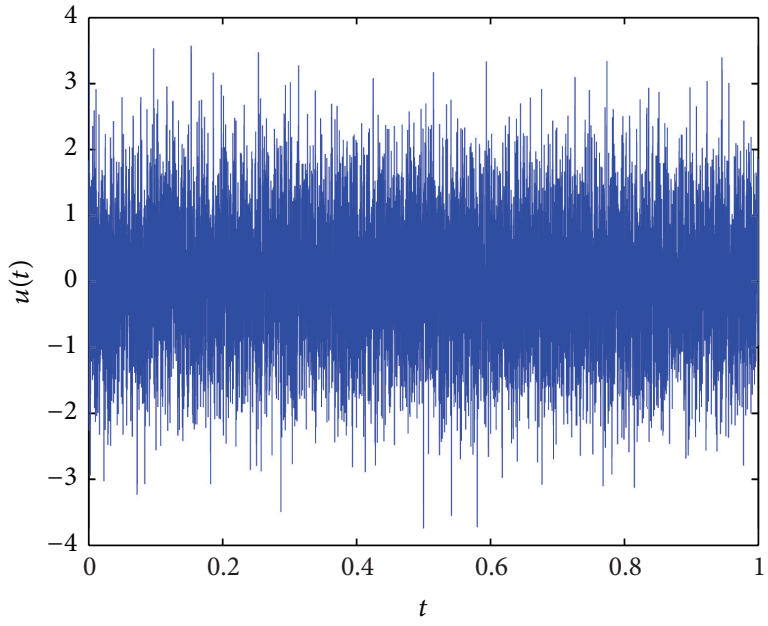

FIgURE 2: The displacement with a Wiener process, the numerical simulation of (60), where $\alpha=1.5, \beta=0.8$.

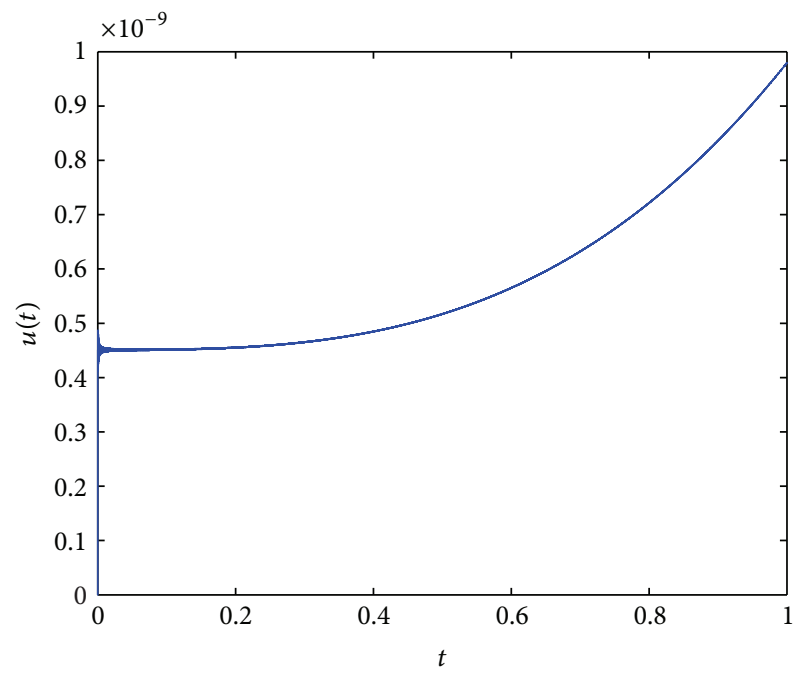

FIGURE 3: The displacement without a Wiener process, the numerical simulation of (63), where $\alpha=1.625, \beta=0.723$.

Letting $p=0.9, g^{*}=0.05, L=l_{1}+l_{2}=0.01, \alpha_{1}=\beta_{1}=\alpha_{2}=$ $\beta_{2}=1, \eta=1$, and $g_{1}(u)=g_{2}(u)=0$, we have

$$
\begin{aligned}
& \alpha \beta_{2}\left[\left(\alpha_{1}+\beta_{1}\right)\right.\left.\eta+\beta_{1}\right]=4.875 \neq \beta_{1}\left(\alpha_{2}+\beta_{2}\right)=2, \\
& \Lambda \triangleq \max \{ \Upsilon_{1}+(\eta+1) \\
& \times\left(\alpha \beta_{2} L+\beta_{1} \Lambda_{2} L+\beta_{1} \beta_{2} \Lambda_{2} \Upsilon_{2}\right) \\
&+\beta_{1}\left(\alpha_{2}+\beta_{2}\right) \Lambda_{2} \Upsilon_{1}, \Upsilon_{2} \\
&+\left(\alpha_{2}+\beta_{2}\right)\left(\alpha \Lambda_{2} L+\alpha \beta_{1} \Lambda_{2} \Upsilon_{1}\right) \\
&\left.+\alpha \Lambda_{1} \Lambda_{2} L+\beta_{2} \beta_{1} \Lambda_{2} \Upsilon_{2}\right\} \\
&=\max \{0.8257,0.9842\}=0.9842<1 .
\end{aligned}
$$

Thus, by Theorem 10, we can get that the problem (63) has at most one solution.

With the above algorithm we get Figures 3 and 4 . 


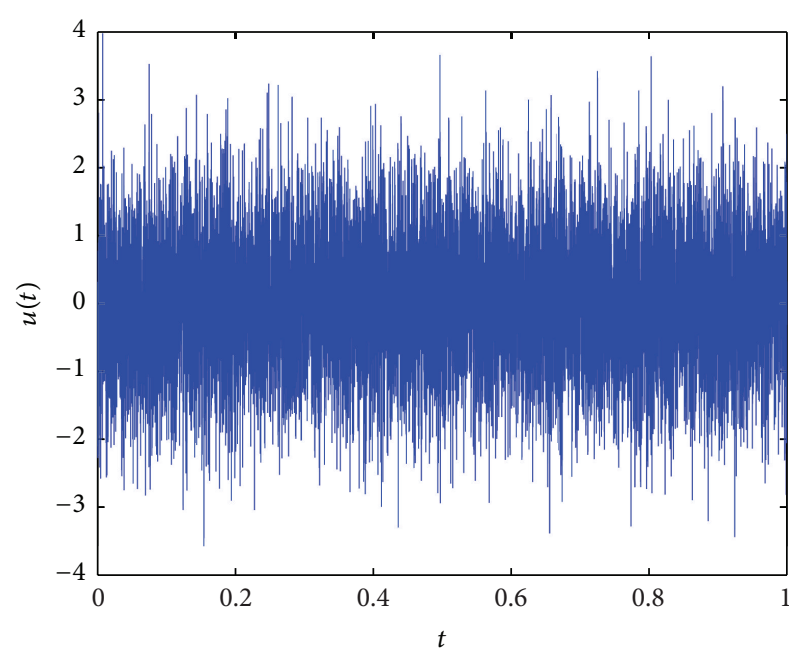

FIGURE 4: The displacement with a Wiener process, the numerical simulation of (63), where $\alpha=1.625, \beta=0.723$.

\section{Acknowledgments}

This project is supported by NNSF of China (Grants nos. 11271087 and 61263006) and Guangxi Scientific Experimental (China-ASEAN Research) Centre no. 20120116.

\section{References}

[1] A. A. Kilbas, H. M. Srivastava, and J. J. Trujillo, Theory and Applications of Fractional Differential Equations, vol. 204 of North-Holland Mathematics Studies, Elsevier Science B.V., Amsterdam, The Netherlands, 2006.

[2] Z. Liu and X. Li, "Existence and uniqueness of solutions for the nonlinear impulsive fractional differential equations," Communications in Nonlinear Science and Numerical Simulation, vol. 18, pp. 1362-1373, 2013.

[3] Z. Liu and X. Li, "On the controllability of impulsive fractional evolution inclusions in Banach spaces," Journal of Optimization Theory and Applications, vol. 156, no. 1, pp. 167-182, 2013.

[4] Z. Liu and J. Sun, "Nonlinear boundary value problems of fractional functional integro-differential equations," Computers \& Mathematics with Applications, vol. 64, no. 10, pp. 3228-3234, 2012.

[5] Z. Liu and J. Sun, "Nonlinear boundary value problems of fractional differential systems," Computers \& Mathematics with Applications, vol. 64, no. 4, pp. 463-475, 2012.

[6] I. Podlubny, Fractional Differential Equations, Academic Press, San Diego, Calif, USA, 1999.

[7] B. Ross, Fractional Calculus and Its Applications, vol. 457, Springer, Berlin, Germany, 1975.

[8] Z. Bai, "On positive solutions of a nonlocal fractional boundary value problem," Nonlinear Analysis. Theory, Methods \& Applications A, vol. 72, no. 2, pp. 916-924, 2010.

[9] M. Benchohra, J. R. Graef, and S. Hamani, "Existence results for boundary value problems with non-linear fractional differential equations," Applicable Analysis, vol. 87, no. 7, pp. 851-863, 2008.

[10] Z. Bai and H. Lü, "Positive solutions for boundary value problem of nonlinear fractional differential equation," Journal of Mathematical Analysis and Applications, vol. 311, no. 2, pp. 495-505, 2005.

[11] V. Daftardar-Gejji, "Positive solutions of a system of nonautonomous fractional differential equations," Journal of Mathematical Analysis and Applications, vol. 302, no. 1, pp. 56-64, 2005.

[12] D. Jiang and C. Yuan, "The positive properties of the Green function for Dirichlet-type boundary value problems of nonlinear fractional differential equations and its application," Nonlinear Analysis. Theory, Methods \& Applications A, vol. 72, no. 2, pp. 710-719, 2010.

[13] E. R. Kaufmann and E. Mboumi, "Positive solutions of a boundary value problem for a nonlinear fractional differential equation," Electronic Journal of Qualitative Theory of Differential Equations, vol. 3, pp. 1-11, 2008.

[14] Z. Liu, "Anti-periodic solutions to nonlinear evolution equations," Journal of Functional Analysis, vol. 258, no. 6, pp. 20262033, 2010.

[15] Z. Liu and D. Motreanu, "A class of variational-hemivariational inequalities of elliptic type," Nonlinearity, vol. 23, no. 7, pp. 17411752, 2010.

[16] Z. Liu, "Existence results for quasilinear parabolic hemivariational inequalities," Journal of Differential Equations, vol. 244, no. 6, pp. 1395-1409, 2008.

[17] C. F. Li, X. N. Luo, and Y. Zhou, "Existence of positive solutions of the boundary value problem for nonlinear fractional differential equations," Computers \& Mathematics with Applications, vol. 59, no. 3, pp. 1363-1375, 2010.

[18] S. Zhang, "Positive solutions for boundary-value problems of nonlinear fractional differential equations," Electronic Journal of Differential Equations, vol. 2006, pp. 1-12, 2006.

[19] M. Benchohra, S. Hamani, and S. K. Ntouyas, "Boundary value problems for differential equations with fractional order and nonlocal conditions," Nonlinear Analysis. Theory, Methods \& Applications A, vol. 71, no. 7-8, pp. 2391-2396, 2009.

[20] L. Yang and H. Chen, "Nonlocal boundary value problem for impulsive differential equations of fractional order," Advances in Difference Equations, vol. 2011, Article ID 404917, 16 pages, 2011.

[21] X. Zhang, X. Huang, and Z. Liu, "The existence and uniqueness of mild solutions for impulsive fractional equations with nonlocal conditions and infinite delay," Nonlinear Analysis. Hybrid Systems, vol. 4, no. 4, pp. 775-781, 2010.

[22] W. Zhong and W. Lin, "Nonlocal and multiple-point boundary value problem for fractional differential equations," Computers \& Mathematics with Applications, vol. 59, no. 3, pp. 1345-1351, 2010.

[23] W. T. Coffey, Yu. P. Kalmykov, and J. T. Waldron, The Langevin Equation, World Scientific, Singapore, 2004.

[24] R. M. Mazo, Brownian Motion: Fluctuations, Dynamicsand Applications, Oxford University Press, Oxford, UK, 2002.

[25] N. Wax, Ed., Selected Papers on Noise and Stochastic Processes, Dover, New York, NY, USA, 1954.

[26] R. Zwanzig, Nonequilibrium Statistical Mechanics, Oxford University Press, New York, NY, USA, 2001.

[27] K. S. Fa, "Generalized Langevin equation with fractional derivative and long-time correlation function," Physical Review E, vol. 73, no. 6, Article ID 061104, 2006.

[28] K. S. Fa, "Fractional Langevin equation and Riemann-Liouville fractional derivative," European Physical Journal E, vol. 24, no. 2, pp. 139-143, 2007. 
[29] E. Lutz, "Fractional Langevin equation," Physical Review E, vol. 64, no. 5, Article ID 051106, 2001.

[30] J. M. Porrà, K.-G. Wang, and J. Masoliver, "Generalized Langevin equations: anomalous diffusion and probability distributions," Physical Review E, vol. 53, no. 6, pp. 5872-5881, 1996.

[31] K. G. Wang, "Long-time-correlation effects and biased anomalous diffusion," Physical Review A, vol. 45, no. 2, pp. 833-837, 1992.

[32] K. G. Wang and M. Tokuyama, "Nonequilibrium statistical description of anomalous diffusion," Physica A, vol. 265, no. 3, pp. 341-351, 1999.

[33] B. Bonilla, M. Rivero, L. Rodríguez-Germá, and J. J. Trujillo, "Fractional differential equations as alternative models to nonlinear differential equations," Applied Mathematics and Computation, vol. 187, no. 1, pp. 79-88, 2007.

[34] B. Ahmad, "Existence of solutions for irregular boundary value problems of nonlinear fractional differential equations," Applied Mathematics Letters, vol. 23, no. 4, pp. 390-394, 2010.

[35] B. Ahmad and J. J. Nieto, "Existence of solutions for nonlocal boundary value problems of higher-order nonlinear fractional differential equations," Abstract and Applied Analysis, vol. 2009, Article ID 494720, 9 pages, 2009.

[36] B. Ahmad and J. J. Nieto, "Existence results for a coupled system of nonlinear fractional differential equations with threepoint boundary conditions," Computers \& Mathematics with Applications, vol. 58, no. 9, pp. 1838-1843, 2009.

[37] B. Ahmad and J. J. Nieto, "Solvability of nonlinear Langevin equation involving two fractional orders with Dirichlet boundary conditions," International Journal of Differential Equations, vol. 2010, Article ID 649486, 10 pages, 2010.

[38] D. Băleanu, R. P. Agarwal, O. G. Mustafa, and M. Coşulschi, "Asymptotic integration of some nonlinear differential equations with fractional time derivative," Journal of Physics A, vol. 44, no. 5, Article ID 055203, 2011.

[39] S. Bhalekar, V. Daftardar-Gejji, D. Baleanu, and R. Magin, "Fractional Bloch equation with delay," Computers \& Mathematics with Applications, vol. 61, no. 5, pp. 1355-1365, 2011.

[40] D. Baleanu, A. K. Golmankhaneh, and A. K. Golmankhaneh, "Fractional Nambu mechanics," International Journal of Theoretical Physics, vol. 48, no. 4, pp. 1044-1052, 2009.

[41] D. Băleanu, O. G. Mustafa, and R. P. Agarwal, "An existence result for a superlinear fractional differential equation," Applied Mathematics Letters, vol. 23, no. 9, pp. 1129-1132, 2010.

[42] D. Baleanu and S. I. Vacaru, "Fedosov quantization of fractional Lagrange spaces," International Journal of Theoretical Physics, vol. 50, no. 1, pp. 233-243, 2011.

[43] M. El-Shahed and J. J. Nieto, "Nontrivial solutions for a nonlinear multi-point boundary value problem of fractional order," Computers \& Mathematics with Applications, vol. 59, no. 11, pp. 3438-3443, 2010.

[44] J. J. Nieto, "Maximum principles for fractional differential equations derived from Mittag-Leffler functions," Applied Mathematics Letters, vol. 23, no. 10, pp. 1248-1251, 2010.

[45] M. R. Ubriaco, "Entropies based on fractional calculus," Physics Letters A, vol. 373, no. 30, pp. 2516-2519, 2009.

[46] G. Wang, B. Ahmad, and L. Zhang, "Impulsive anti-periodic boundary value problem for nonlinear differential equations of fractional order," Nonlinear Analysis. Theory, Methods \& Applications A, vol. 74, no. 3, pp. 792-804, 2011.

[47] B. Ahmad, J. J. Nieto, A. Alsaedi, and M. El-Shahed, "A study of nonlinear Langevin equation involving two fractional orders in different intervals," Nonlinear Analysis. Real World Applications, vol. 13, no. 2, pp. 599-606, 2012.

[48] A. Chen and Y. Chen, "Existence of solutions to nonlinear Langevin equation involving two fractional orders with boundary value conditions," Boundary Value Problems, vol. 2011, Article ID 516481, 17 pages, 2011.

[49] B. L. Guo, X. K. Pu, and F. H. Huang, Fractional Partial Differential Equations and Their Numberical Solution, Science Press, Beijing, China, 2011.

[50] P. Guo, C. P. Li, and F. H. Zeng, "Numerical simulation of the fractional Langevin equation," Thermal Science, vol. 16, pp. 357363, 2012.

[51] M. Weilbee, Effcient Numerical Methods For Fractional Differetnial Equations and Their Analytical Background, Thechnical University of Braunschweig, Braunschweig, Germany, 2005.

[52] M. M. Meerschaert and C. Tadjeran, "Finite difference approximations for fractional advection-dispersion flow equations," Journal of Computational and Applied Mathematics, vol. 172, no. 1, pp. 65-77, 2004.

[53] K. B. Oldham and J. Spanier, The Fractional Calculus: Theory and Applications of Differentiation and Integration to Arbitrary Order, Academic Press, Pittsburgh, Pa, USA, 1974. 


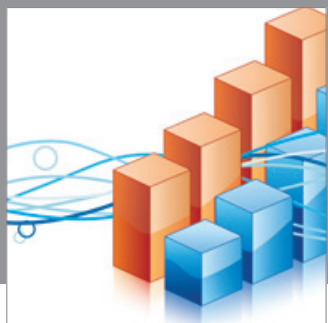

Advances in

Operations Research

mansans

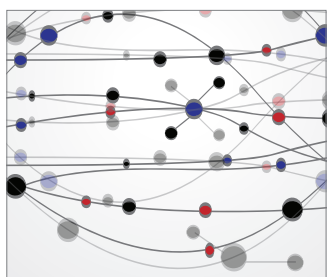

The Scientific World Journal
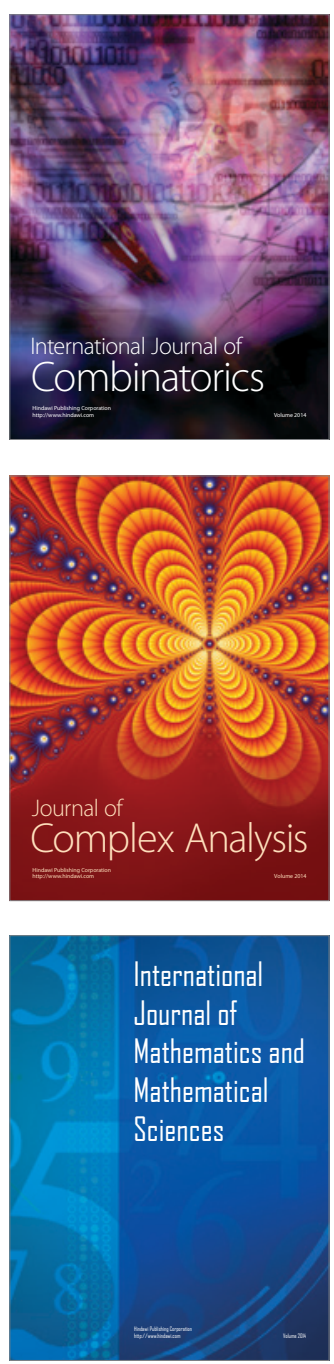
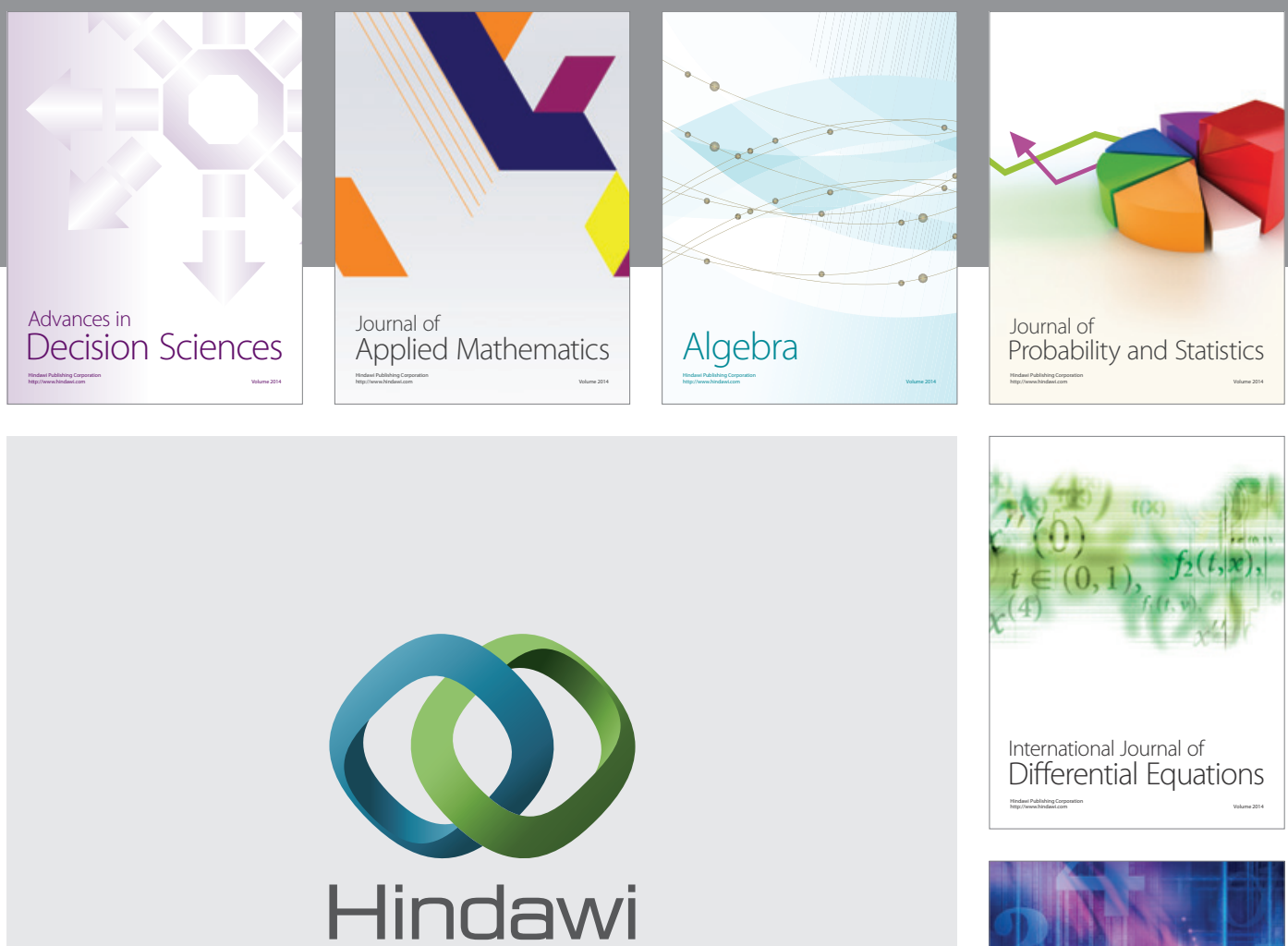

Submit your manuscripts at http://www.hindawi.com
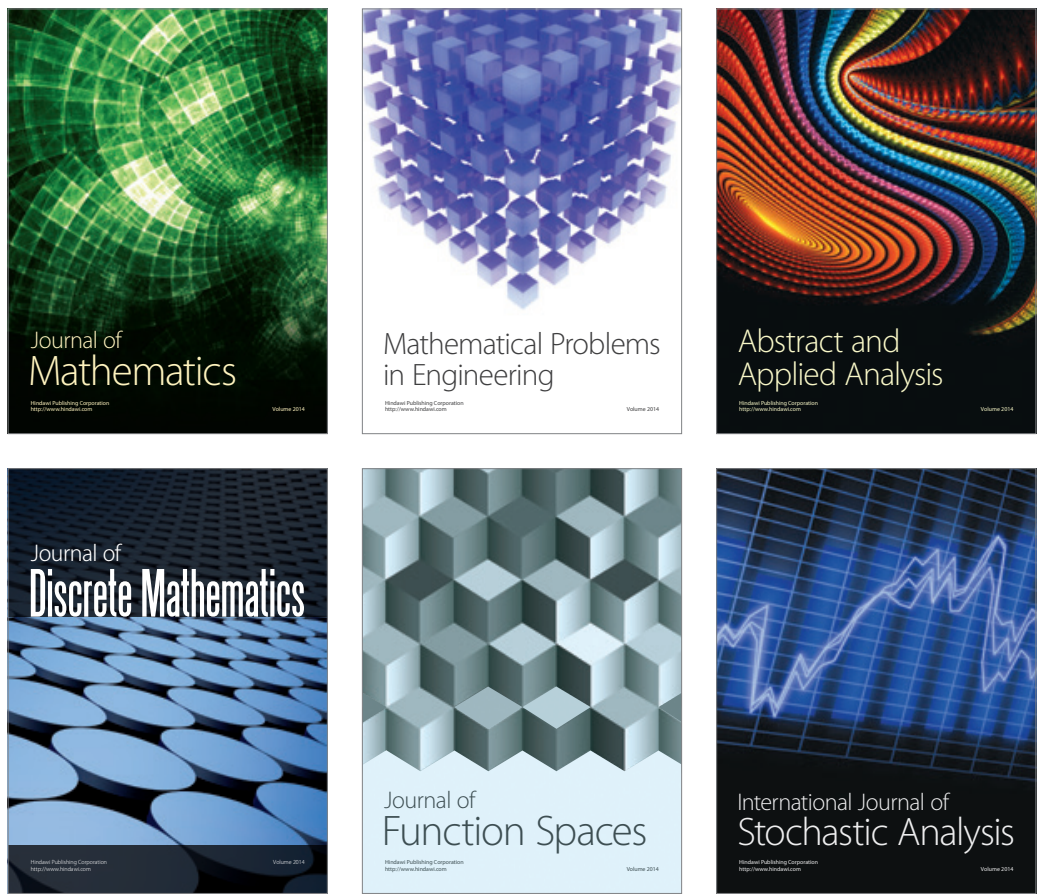

Journal of

Function Spaces

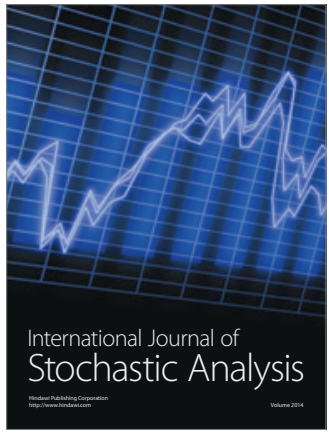

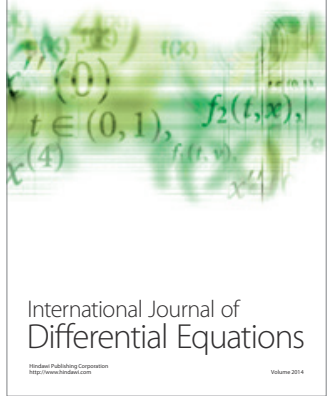
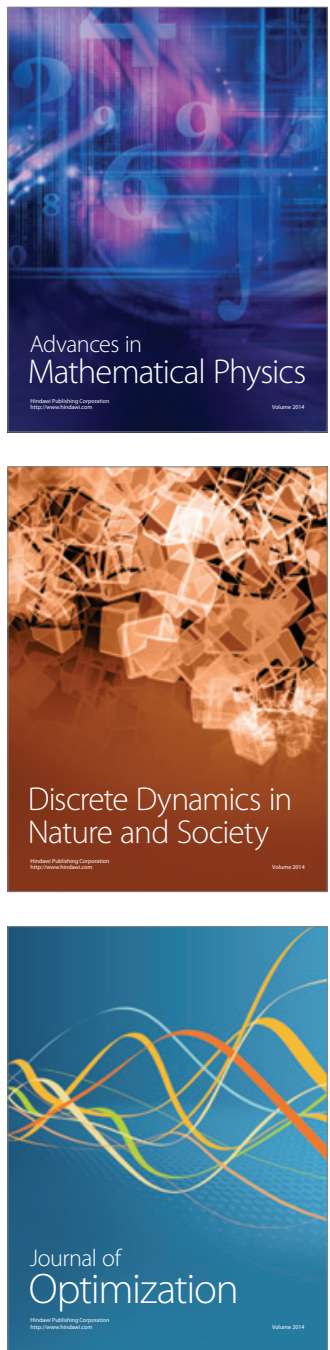